\title{
A Comprehensive Survey on Hierarchical-Based Routing Protocols for Mobile Wireless Sensor Networks: Review, Taxonomy, and Future Directions
}

\author{
Nabil Sabor, ${ }^{1,2}$ Shigenobu Sasaki, ${ }^{1}$ Mohammed Abo-Zahhad, ${ }^{2}$ and Sabah M. Ahmed ${ }^{2}$ \\ ${ }^{1}$ Department of Electrical and Electronic Engineering, Niigata University, Niigata 950-2181, Japan \\ ${ }^{2}$ Electrical and Electronics Engineering Department, Faculty of Engineering, Assiut University, Assiut 71516, Egypt \\ Correspondence should be addressed to Nabil Sabor; nabil_sabor@aun.edu.eg
}

Received 19 June 2016; Accepted 28 August 2016; Published 10 January 2017

Academic Editor: Pierre-Martin Tardif

Copyright (C) 2017 Nabil Sabor et al. This is an open access article distributed under the Creative Commons Attribution License, which permits unrestricted use, distribution, and reproduction in any medium, provided the original work is properly cited.

Introducing mobility to Wireless Sensor Networks (WSNs) puts new challenges particularly in designing of routing protocols. Mobility can be applied to the sensor nodes and/or the sink node in the network. Many routing protocols have been developed to support the mobility of WSNs. These protocols are divided depending on the routing structure into hierarchical-based, flatbased, and location-based routing protocols. However, the hierarchical-based routing protocols outperform the other routing types in saving energy, scalability, and extending lifetime of Mobile WSNs (MWSNs). Selecting an appropriate hierarchical routing protocol for specific applications is an important and difficult task. Therefore, this paper focuses on reviewing some of the recently hierarchical-based routing protocols that are developed in the last five years for MWSNs. This survey divides the hierarchicalbased routing protocols into two broad groups, namely, classical-based and optimized-based routing protocols. Also, we present a detailed classification of the reviewed protocols according to the routing approach, control manner, mobile element, mobility pattern, network architecture, clustering attributes, protocol operation, path establishment, communication paradigm, energy model, protocol objectives, and applications. Moreover, a comparison between the reviewed protocols is investigated in this survey depending on delay, network size, energy-efficiency, and scalability while mentioning the advantages and drawbacks of each protocol. Finally, we summarize and conclude the paper with future directions.

\section{Introduction}

The importance of Mobile Wireless Sensor Networks (MWSNs) in monitoring and collecting data from the environment has been increased in the recent years. A MWSN typically consists of mobile sensor nodes and/or mobile $\operatorname{sink}(\mathrm{s})$ that can move within the network. Mobility can be achieved by attending the mobile elements with mobilizers for controlling their locations or they can be attached to transporters like vehicles, animals, robots, and so forth $[1,2]$. Some real-world applications demand environments composed of static and mobile sensor nodes within the same network, while other applications request complete mobile sensors environment [3-5]. For instance, in outdoor medical applications, patients use body sensor networks (mobile nodes) to monitor their vital signs (e.g., heart rate and blood pressure) which are transmitted to doctor (mobile sink) and monitoring room (static sink) for tracking and monitoring patients' health status, while in wild life applications, sensors are equipped on animals (mobile nodes) to observe their behavior within a spacious habitat which are collected via a mobile sink (e.g., a car or a plane) [6]. The sensor nodes are small devices and can perform many tasks, including event sensing, data processing, and data communication. Each node consists of four main parts as shown in Figure 1. The first part is a sensing unit that senses a phenomenon and converts sensing data into a digital form using a sensor and Analog-to-Digital Converter (ADC). Processor unit is the second part that processes all data and controls operations of the other parties. The third part is a transceiver unit that is used to transmit and receive data with a limited transmission range. Power unit is the last 


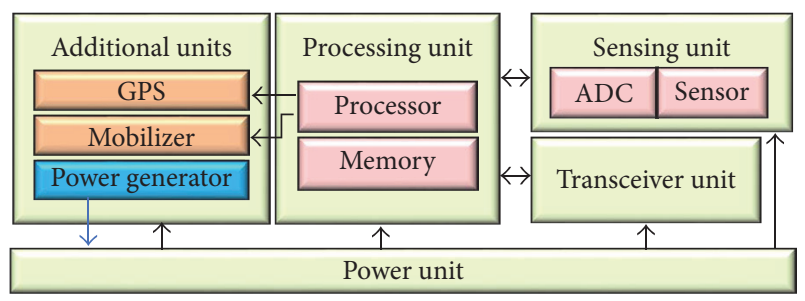

FIGURE 1: Structure of sensor node.

part that supplies power to all parties. Moreover, a sensor node might additionally have some specific components, such as the Global Positioning System (GPS), mobilizer, and power generator units. The GPS unit can help a sensor node to obtain its own location information while the mobilizer unit offers the movement capability of a sensor node. The power generator unit is responsible for power generation by applying some specific techniques such as solar cell [7].

A sensor node has limited on-board storage, processing, power, and radio capabilities due to its small size. Therefore, MWSNs require effective mechanisms to utilize and resolve the limited resources. Routing is one of these mechanisms that prolongs the lifetime of network by reducing the energy consumption in communication. The routing protocol should consider the network structure, data sending methods, node and link heterogeneity, node mobility, consumption energy, coverage, connectivity, data aggregation, and quality of service issues in order to be an efficient and reliable protocol. The developed routing protocols can be grouped based on the routing structure into hierarchical-based, flat-based, and location-based routing protocols. According to literature [8-16], the hierarchicalbased routing protocols outperform the other routing types in saving energy, extending a lifetime of WSNs, and scalability. The hierarchical-based routing partitions the network into multiple groups. Each group contains one head node and many member nodes (MNs). MN only senses and delivers its sensed data to its related head node, while a head is responsible for collecting and aggregating data of its MNs and then transfers the aggregated data to sink.

Hierarchical-based routing is a feasible solution for reducing energy consumption in WSNs due to reduction of redundant data transmission. In addition, it can effectively balance the load among the sensor nodes via assigning different tasks for each sensor node according to its capabilities. Finally, it can easily achieve collision-free network by using a proper MAC protocol. Based on the approach of data routing, the hierarchical-based routing can be broadly divided into classical hierarchical-based routing and optimized hierarchical-based routing. This survey will specialize in reviewing some of the recently classical and optimized hierarchical-based routing protocols that are developed in the last five years for the MWSNs. Also, a detailed classification of the reviewed protocols is presented in this survey based on many different metrics. These metrics are routing approach, control manner, mobile element, mobility pattern, network architecture, clustering attributes, protocol operation, path establishment, communication paradigm, energy model, protocol objectives, and applications. Moreover, we compare between the surveyed protocols on the basis of delay, network size, energy-efficiency, and scalability while analyzing the advantages and drawbacks of each protocol.

The paper is organized as follows. Section 2 is a literature survey about various clustering protocols of MWSNs. Taxonomy criteria of the hierarchical-based routing protocols are explained in Section 3. Section 4 discusses the metrics that are used for evaluation of the hierarchical-based routing protocols. Reviewing the hierarchical-based routing protocols of MWSNs is given in Section 5. In Section 6, the classification and comparison of the reviewed protocols are described. Section 7 offers some conclusions and future directions.

\section{Related Works}

This section summarizes the previous surveys [8-16] of the routing protocols that are designed for MWSNs in literature. A survey of routing techniques that are developed for wireless ad-hoc or WSN networks was presented in [8]. The aim of this survey is to identify the routing protocol that can support the mobility of the sensor nodes in mixed WSNs, which consist of both static and mobile sensor nodes. Moreover, authors highlighted the advantages/disadvantages and performance issues of each routing technique. Authors in [9] gave a comprehensive taxonomy of the routing protocols based on discovery, data transfer, routing, and motion control. This survey classified the routing protocols into flat routing and proxy-based routing. In [10], authors reviewed LEACH-M, LEACH-ME, CBR-M, ECBR-MWSN, E2R2, 2L-LEACH-M, FTCPMWSN, LFCP-MWSN protocols that are developed for the MWSNs. In addition, they presented a comparison between the reviewed protocols based on assumptions, $\mathrm{CH}$ selection, location awareness, scalability, and complexity. In [11], the authors categorized the flat- and hierarchicalbased routing protocols of MWSNs depending on their network structure, state of information, energy-efficiency, and mobility. This classification summarizes the advantages and disadvantages of the reviewed routing protocols. Moreover, this survey gives an insight into the enhancements that can be done to improve the existing routing protocols. A survey of the location-based routing protocols that support the sink mobility is presented in [12]. This survey classified the location-based routing approaches into backbone-based and rendezvous-based approaches according to their network structures while highlighting the pros and cons of each type.

A survey in [13] classified the existing routing protocols of MWSNs into delay-sensitive routing protocols and delaytolerant routing protocols based on delay, into centralized and distributed protocols based on the routing decisions, into discretely and continuously based routing protocols based on mobility pattern, and into location-based and topology-based protocols depending on the routing information needed. Also, this survey explained how each protocol works and mentioned the advantages and disadvantages of each one. Authors in [14] surveyed the distributed routing protocols that support mobile sinks. In addition, they explained the challenges and the design requirements of a mobile sinkbased routing protocol. The surveyed protocols are compared 
based on position awareness, sink mobility pattern, virtual structure type, data aggregation, multisink support, protocol overhead, structure accessibility, and hotspot mitigation. A survey of eleven mobile sink-based routing protocols was adopted in [15]. These protocols are compared based on number of mobile sinks, type of protocol, and sink mobility pattern. In [16], a brief overview of the cluster-based protocols for static WSNs, where the sensor nodes are fixed, and for MWSNs, where some or all the sensor nodes are mobile in nature, was presented. Moreover, the surveyed protocols are compared based on the basic assumptions, working environment, advantages, limitations, and working style.

As shown in the previous discussion, there are no works focusing on reviewing the hierarchical routing protocols that support the mobility of sensor nodes and/or sink node. Therefore, this survey paper will provide a comprehensive review and fine-grained classification of the recently hierarchical-based routing protocols that were developed in the last five years for MWSNs. The reviewed protocols are classified based on routing approach, control manner, mobile element, mobility pattern, network architecture, clustering attributes, protocol operation, path establishment, communication paradigm, energy model, protocol objectives, and applications. Based on delay, network size, energy-efficiency, and scalability, a comprehensive comparison between the surveyed protocols will be considered in this paper while analyzing the advantages and drawbacks of each protocol.

\section{Taxonomy Criteria of Hierarchical-Based Routing Protocols}

The main aim of hierarchical-based routing in MWSNs is saving the residual energy of each sensor node, extending the network lifetime, and ensuring the connectivity among the sensor nodes. Here, we present a detailed taxonomy and classification of the hierarchical-based routing protocols based on different metrics. These metrics are routing approach, control manner, mobile element, mobility pattern, network architecture, clustering attributes, protocol operation, path establishment, communication paradigm, energy model, protocol objectives, and applications as shown in Figure 2.

3.1. Routing Approach. The challenge of data routing can have two broad methods, namely, classical-based method and optimized-based method.

(a) Classical-Based Routing. In the classical-based routing, head nodes are selected randomly using the timer function, which causes an uneven traffic flow in different head nodes. Although they are suitable for applications of WSNs, they still have many challenges such as scalability, load balancing, connectivity, coverage, and robustness.

(b) Optimized-Based Routing. Routing in WSNs puts new challenges for researchers as various classical protocols lack fault tolerance, energy-efficiency, connectivity, robustness, and scalability. Researchers have developed some robust routing protocols based on optimization algorithms such as Particle Swarm Optimization (PSO), Fuzzy Logic (FL), Genetic Algorithm (GA), and Artificial Bee Colony (ABC), which provide optimal solutions to the mentioned problems. Thus in the optimized-based routing, the head nodes are determined based on multicriteria to achieve the requirements of QoS.

3.2. Control Manner. On the basis of control manner, routing approaches of MWSNs can be centralized, distributed, or hybrid approaches.

(a) Centralized Approaches. In the centralized approaches, a sink/head node requires global information (e.g., energy level, geographical position) of the network/group to control the network/group. These approaches are used to organize the network into clusters and assign a head node for each one.

(b) Distributed Approaches. In the distributed approaches, the sensor nodes collaborate with each other to build routes without need for the global information of the network. Each sensor node can execute its own algorithm and takes the decision of becoming a head node or not. These approaches are used for coordination between the head nodes.

(c) Hybrid Approaches. Hybrid approaches combine the features of being centralized and distributed.

3.3. Mobile Element. Since the network consists of a large number of sensor nodes and sink nodes, the mobility can apply to the sensor nodes and/or the sink node(s) depending on the applications. Therefore, the routing protocols can be classified based on the mobile elements into protocols supporting sink node(s) mobility, protocols supporting sensor nodes, and protocols supporting mobility of both sensor nodes and sink node(s).

3.4. Mobility Pattern. One of the main challenges in routing of MWSNs is determining the moving pattern of the mobile element (i.e., sensor nodes or sink node). Depending on the application and network size, there are different mobility patterns.

(a) Predefined Mobility Pattern. In this pattern, the mobile element moves along a predefined path within the sensor field and stops at predefined positions to perform a specific task. This pattern can be used for the mobile sink.

(b) Random Mobility Pattern. The random mobility pattern can be used for the mobile sensor nodes and the mobile sink. In this pattern, the mobile element moves randomly within the sensor field. The synthetic mobility models are used for simulating the random mobility of a mobile element such as Random WayPoint mobility model (RWP) and Reference Point Group Mobility Model (RPGM) [17-19].

(c) Controlled Mobility Pattern. In the controlled mobility, the mobile element is guided based on the control of the routing protocol. The movement of the mobile element is controlled based on some factors such as energy level, avoiding energy 


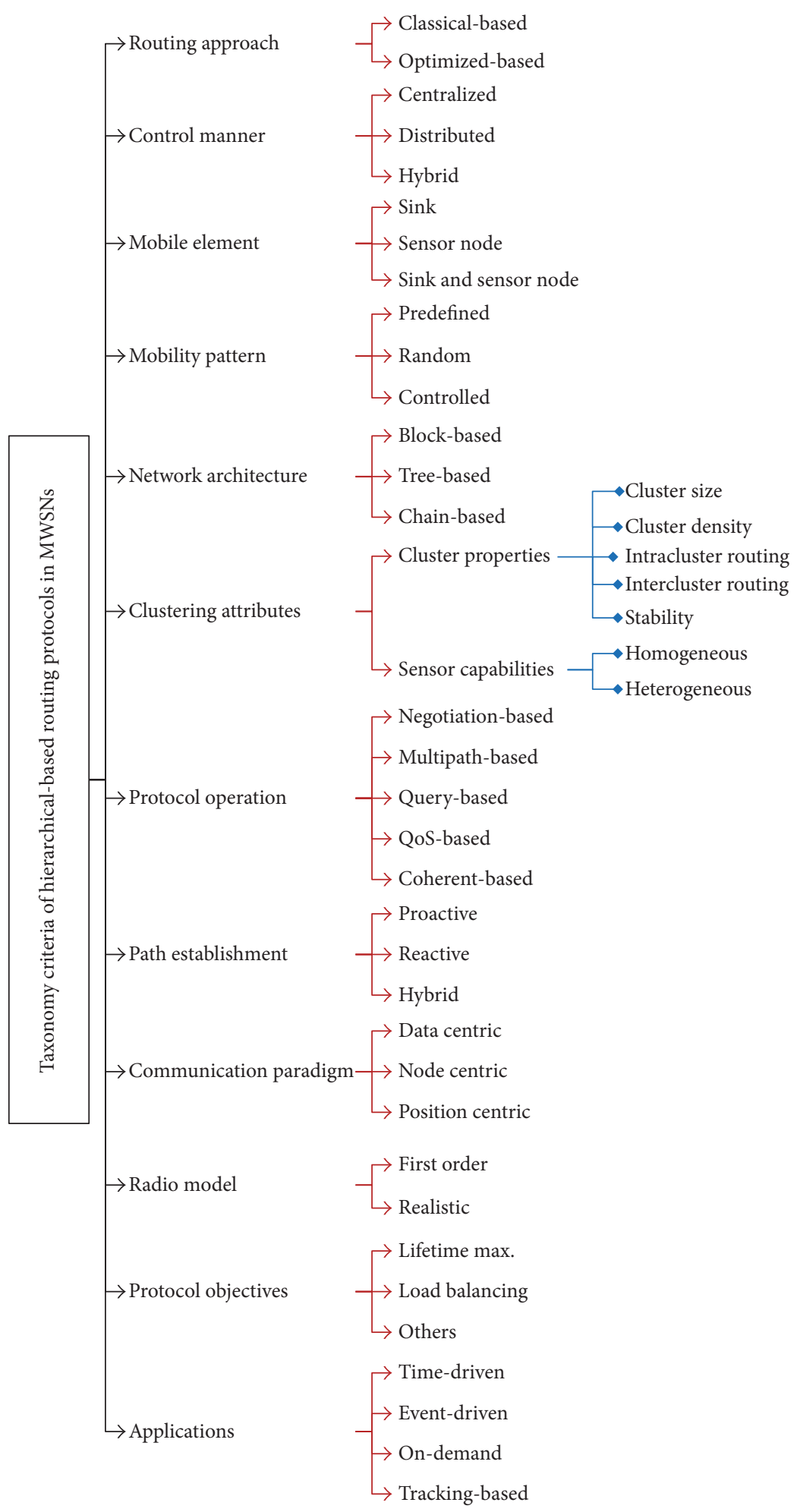

FIGURE 2: Taxonomy criteria of hierarchical-based routing protocols in MWSNs.

hole or hotspot problem [20], and connectivity, to achieve better results.

3.5. Network Architecture. The underlying network architecture plays an important role in the function of the hierarchical routing protocols in MWSNs. Based on the network architecture, the hierarchal routing protocols can be classified into three categories, which are block-based hierarchical routing, tree-based hierarchical routing, and chain-based hierarchical routing.

(a) Block-Based Hierarchical Routing. In the block-based hierarchical routing protocols, the network is divided into 
groups called clusters. Each one has a head node called Cluster Head $(\mathrm{CH})$ node that is responsible for collecting and aggregating the data of its MNs and then transfers the aggregated data to the sink node. The main problem with these protocols is how to select $\mathrm{CH}$ and the limited range of sensor nodes to connect directly with sink.

(b) Tree-Based Hierarchal Routing. In the tree-based hierarchical routing protocols, a routing tree is formed among all sensor nodes and the sink is the root of this tree. Leaf nodes in the routing tree send data to their parent. Each parent node aggregates the received data and sends it to the next level parent node towards the sink.

(c) Chain-Based Hierarchical Routing. In the chain-based hierarchical routing protocols, one or more chains are constructed to connect the nodes for data transmission. A head node for each chain called leader is chosen to collect data from the chain members. Data is delivered from the farthest node from sink along the chain until the leader node forwards the final packet toward the sink. However, the data packet reaches the sink via a large number of hops, which increases the packet delay. Moreover, the chain-based routing has less ability of robustness because the failure of one node breaks the chain and data will be dropped.

3.6. Clustering Attributes. The attributes of the clustering process have an important effect on the performance of hierarchical-based routing. The cluster properties and the sensor capabilities are the two main issues in the clustering attributes [21, 22].

3.6.1. Cluster Properties. In the hierarchical-based routing protocols, the characteristics of the formed clusters are used to differentiate between these clusters in terms of saving energy, load balancing, and lifetime.

(a) Cluster Size. From the point of cluster size, the hierarchical-based routing protocols in MWSNs can be grouped into equal and unequal clustering. In the equal clustering protocols, all clusters have the same size, while in the unequal clustering protocols, clusters have different sizes. In general, unequal clustering protocols are used to balance the load among the nodes and solve the energy hole problem.

(b) Cluster Density. Cluster density is the number of cluster members. Density of cluster affects the energy consumption of $\mathrm{CH}$. Clustering protocols can be static clustering protocols and dynamic clustering protocols. In the former one, clusters have fixed density, but the cluster density in the second one is variable.

(c) Intracluster Routing. Intracluster routing is the communication between MNs and $\mathrm{CH}$. This communication can be a single-hop or a multihop routing. In the single-hop routing, MNs directly deliver their data to $\mathrm{CH}$. However in the multihop routing, member nodes transmit their data to $\mathrm{CH}$ via relay nodes. (d) Intercluster Routing. Intercluster routing is the communication between the sensor nodes/CHs and sink node. The intercluster communication can be a single-hop or a multihop routing. In the single-hop routing, sensor nodes/CHs send their data directly to sink. In contrast to it, sensor nodes/CHs transmit their data to sink using intermediate nodes in the multihop routing.

(e) Stability. The stability of routing process depends on the cluster density. If the cluster density is fixed, the stability of routing is said to be fixed. Otherwise, the routing stability is considered variable because the cluster density varies throughout the routing process.

3.6.2. Sensor Capabilities. Based on the resources of the sensor nodes, MWSNs can be classified into homogeneous and heterogeneous networks.

(a) Homogeneous Network. In the homogenous network, all sensor nodes have the same energy, computation, and communication resources. In this type of networks, $\mathrm{CHs}$ are assigned according to a random manner or other criteria.

(b) Heterogeneous Network. In the heterogeneous network, sensor nodes have unequal capabilities in heterogeneous environment. Therefore, the role of $\mathrm{CHs}$ is specified to sensor nodes that have more capabilities.

3.7. Protocol Operation. Depending on the protocol operation, the hierarchical-based routing protocols are divided into negotiation-based, query-based, multipath-based, coherentbased, and QoS-based routing.

(a) Negotiation-Based Routing. In this type of routing, a high level of descriptors is utilized for the negotiation between the sensor nodes to minimize the duplicated information and avoid the redundant data. Generally, this negotiation should be done before real data transmission between the source and the relay node or the sink node.

(b) Query-Based Routing. This type of routing depends upon queries from a destination. The source node transmits its data in response to the received query from the destination node.

(c) Multipath-Based Routing. In the multipath routing, multiple paths are constructed between a source and a destination to increase the fault tolerance and enhance the network performance.

(d) Coherent-Based Routing. Different data processing mechanisms are presented to save the processing computations that consume a significant part of the node energy. Coherent and noncoherent are the two main data processing approaches that are used to save the consumption energy in data computations. In the noncoherent data processing technique, the sensor node processes the data locally and then forwards it to the aggregator. Aggregator is a node, which aggregates the received data from many sensor nodes and forwards the aggregated packets to sink. In the coherent method, a sensor node performs minimum data processing 
and sends data to the aggregator. After receiving the data, the aggregator is responsible for the major and complex part of processing.

(e) QoS-Based Routing. The used algorithm in this type of routing ensures the QoS requirements of the data. These requirements can be reliability, delay, or bandwidth. The task of routing protocol is balancing the dissipated energy while achieving the QoS conditions.

3.8. Path Establishment. The path establishment mechanism is responsible for identifying or discovering routes from a source to the intended receiver. This mechanism can also be used to distinguish between different types of the hierarchical-based routing protocols.

(a) Proactive Routing. This type of routing is also often described as table-driven, because each node selects the best path and forwards its data based on the contents of a routing table. This table contains a list of paths between a node and one or more next-hop neighbors and also cost associated with each path. In this type of network, nodes periodically switch on their sensors and radios to sense the data and transmit it to the destination via a certain route from the routing table. This routing type is suitable for periodic data monitoring applications like collecting data about temperature change over a particular area.

(b) Reactive Routing. In the reactive routing, the node reacts immediately to sudden changes in the sensing event and does not already have a route established. This type of routing adds some delay for discovering the route before transmitting the data. Also, it is well suited for time-critical applications, for example, explosion detection and intrusion detection.

(c) Hybrid Routing. In this routing type, nodes not only react to sudden changes in the sensing event but also send their data at periodic intervals in an efficient method to the destination.

3.9. Communication Paradigm. The communication between the sink and the sensor nodes can be node centric, data centric, or location centric.

(a) Node Centric. In the node-centric protocols, destinations are specified using numerical addresses (or identifiers) of nodes. In this type, sensor node can forward its data to specific destination via its ID.

(b) Data Centric. In the data centric technique, sink forwards queries to a particular area within a sensor field and waits for data of the sensors that are located in the selected region. The source sensors of the selected region send their data to the sink via intermediate nodes. These intermediate sensor nodes aggregate the collected data from multiple sources and forward the aggregated packets to the sink. This process saves the dissipated energy in the redundant data.

(c) Location Centric. In the location-centric routing, the sensor nodes should know their locations in the sensor field.
Location information is used to construct best routing, which enhances the network performance.

3.10. Radio Model. The major task of the hierarchical-based routing protocols is saving the residual energy of each sensor node and extending the network lifetime. Since the energy consumed by the radio of the sensor node represents the largest portion of the consumed energy [23], the routing protocols can be surveyed according to the model of the sensor radio. The radio of the sensor node is simulated as the first-order model [24] or as the realistic radio model such as CC2420 [25].

3.11. Protocol Objectives. The hierarchical-based routing protocols have been developed to save the dissipated energy and extend the lifetime of MWSNs. Accordingly, the hierarchicalbased routing protocols of MWSNs can be classified according to the above criteria based on different objectives. These objectives can be data aggregation, load balancing, lifetime maximization, stability period extension, guarantee of connectivity, fault tolerance, avoiding hotspot, and so forth. The routing protocol can develop to achieve one or more objectives at the same time.

3.12. Applications. Since there is not a routing protocol appropriate for all applications, this survey specifies the suitable applications for each hierarchical routing protocol. Applications of MWSNs can broadly be split into eventdriven, time-driven, on-demand, and tracking-based applications [26].

(a) Event-Driven Applications. Sensor nodes deployed for such type of applications are expected to be inactive most of the time and bursting into activity when an event is detected. Then, the detected event is reported to the sink. This type of application can be found in forest fires, grass fires, volcanic eruptions, and so forth.

(b) Time-Driven Applications. In this type of applications, each sensor is expected to constantly produce some amount of data, which has to be conveyed periodically to the sink. The time-driven applications include monitoring the environmental conditions like affecting crops, temperature, humidity, and lighting.

(c) On-Demand Applications. In some applications, the sink is not interested in data updates from all the nodes in the network. This is done via sending queries to a set of sensor nodes at different times from different regions. This results in a more energy-efficient use of resources.

(d) Tracking-Based Applications. This class is helpful when the source of an event is mobile. The sensor nodes can report the event source's position to the sink, potentially with estimates about speed and direction. The tracking applications combine some of the above three classes. This class can be used in the military application (e.g., tracking an intruder), the environmental applications (e.g., tracking the movements and patterns of insects and birds), and the intelligent applications (e.g., tracking of vehicles). 


\section{Evaluation Metrics of Hierarchical Routing Protocols}

In this survey, the various hierarchical routing protocols will be evaluated based on delay, network size, energy-efficiency, and scalability.

(a) Delay. Delay is a time of transmitting a packet from the source to the sink. This delay provides a close approximation for the actual latency. Delay is an important factor for real time applications.

(b) Network Size. Each hierarchical routing protocol is suitable for a certain kind of network, such as large-scale networks or small-scale networks. Surely, there are hierarchal routing protocols that perform well in both large-scale and small-scale networks.

(c) Energy-Efficiency. Due to the limited energy of sensor nodes in MWSNs, most of the hierarchical routing protocols are developed to save the energy of sensors and extend the network lifetime. Thus, the energy-efficiency is the main objective of the routing protocols.

(d) Scalability. As the sensors are becoming ever cheaper, unlike the traditional networks, WSNs are becoming larger up to tens of thousands of nodes. Therefore, a routing protocol should be scalable for adding new sensor nodes.

\section{Hierarchical-Based Routing Protocols of MWSNs}

In hierarchical-based routing protocols, sensor nodes perform different tasks such as sensing, processing, transmitting, and receiving. Some of these sensor nodes, that are called $\mathrm{CHs}$ or leaders, are responsible for collecting and processing data and then forwarding it to sink, while the task of other nodes, which are called MNs, is sensing the sensor field and transmitting the sensing data to the head nodes. Hierarchicalbased routing is a two-layer architecture, where head nodes selection is performed in the first layer and the second layer is responsible for routing. The developed hierarchical routing protocols could be classified into two classes, classical-based routing and optimized-based routing.

5.1. Classical Hierarchical-Based Routing Protocols. In the classical hierarchical-based routing protocols, head nodes are selected only based on generated random values. If the random value is less than a certain threshold, the node will be a head. Due to the random selection, an uneven traffic flow in different head nodes may occur. Thus, some head nodes will exhaust energy, and the performance of the network is affected. Here, we review some of the recently classical hierarchical-based routing protocols that are developed for the MWSNs in the last five years.

5.1.1. LEACH-Mobile Protocol. Authors in [27] developed a routing protocol called LEACH-Mobile to support mobility of the sensor nodes. LEACH-Mobile confirms whether a mobile node is capable of communicating with its Cluster Head $(\mathrm{CH})$ within the timeslot allotted in TDMA schedule. The node that does not receive a request from its $\mathrm{CH}$ during two successive TDMA schedules can identify that it is out of its cluster as illustrated in Figure 3. The moved node can join a new cluster by broadcasting a cluster joint request message for which it will receive a cluster joint ACK message from the new $\mathrm{CH}$. However, LEACH-Mobile assumes that the $\mathrm{CH}$ are stationary. Hence, LEACH-Mobile is not efficient in terms of dissipated energy and data delivery rate because moving $\mathrm{CH}$ will lead to dropping a large number of packets.

5.1.2. LEACH-Mobile-Enhanced Protocol. To alleviate the problem of LEACH-Mobile, an enhanced version of LEACHMobile called LEACH-Mobile-Enhanced (LEACH-ME) was developed in [28]. LEACH-ME selects a node with the lowest mobility factor to be $\mathrm{CH}$. Mobility factor is determined based on the number of nodes' movement outside the cluster. More elaborately, the mobility factor is calculated in an extra timeslot in each frame via multiplying node's velocity with the time required for a node to move from a position to another. However, LEACH-ME consumes much energy for calculating the mobility factor of each node during the extra slot in each frame.

5.1.3. Mobile Sink-Based Routing Protocol. A mobile sinkbased routing protocol (MSRP) [29] was designed to prolong the network lifetime and alleviate the hotspot or the energy hole problem of WSNs. MSRP utilizes a mobile sink that moves based on the residual energy of $\mathrm{CH}$ s for data gathering in the clustered network. The operation of MSRP consists of two main phases which are a setup phase and a steady-state phase. In the setup phase, the network is partitioned into clusters and the mobile sink advertises its location to $\mathrm{CHs}$ by broadcasting beacon message for registration, while in the steady-state phase, the mobile sink sends a TDMA schedule to the registered $\mathrm{CH}$. Then $\mathrm{CH}$ s collect data of $\mathrm{MNs}$ via a single- or a multihop communication. After collecting data, the mobile sink moves to a new place based on the residual energy of CHs. Simulation results cleared that the MSRP balances the energy consumption among the nodes and solves the hot spot problem due to changing the neighbors of the mobile sink.

5.1.4. Cluster-Based Routing Protocol. Mobility and traffic adapted cluster-based routing protocol for MWSNs (CBRMobile) [30] was designed to collect data from the mobile sensor nodes with maximum delivery ratio and minimum average delay. The mobility and traffic adapted scheduling based MAC design enables CHs to reuse the free or unused timeslots to support the mobility of sensor nodes. Each $\mathrm{CH}$ maintains two simple database tables for mobility and traffic to achieve this adaptation as illustrated in Figure 4. The designed CBR-Mobile protocol enables the disconnected mobile sensor nodes (MSNs) to rejoin the network through other $\mathrm{CH}$ s within a short time. As a result, the CBR-Mobile protocol can significantly reduce the packet loss compared to the LEACH-Mobile protocol. However, the control overhead used in the CBR-Mobile protocol is higher than that in the 

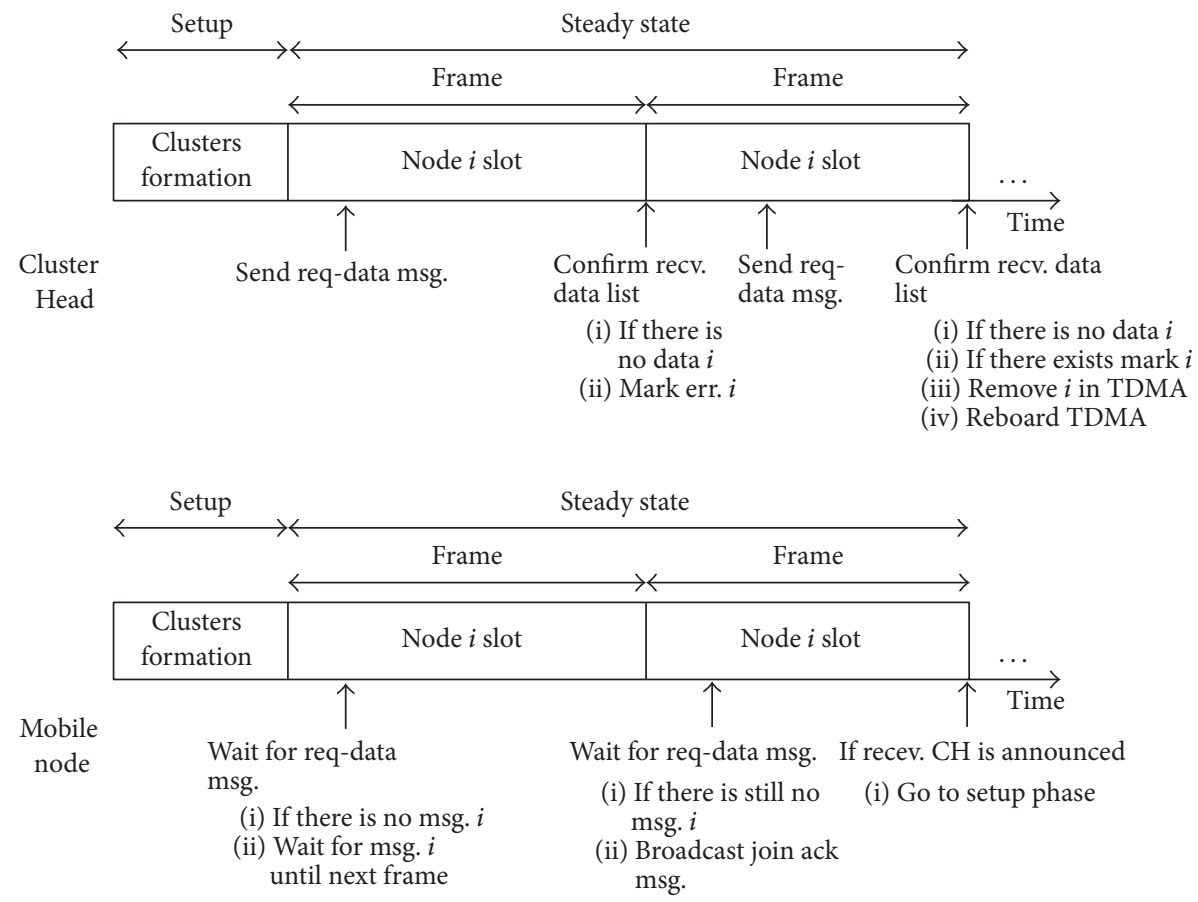

Figure 3: LEACH-Mobile protocol.

LEACH-Mobile, which may cause more energy consumption and reduce the network lifetime.

5.1.5. Mobility-Based Clustering Protocol. Authors in [31] developed a Mobility-Based Clustering protocol (MBC) to improve the performance of MWSNs. The operation of MBC consists of a setup phase and a steady-state phase in each round. In the setup phase, a sensor node elects itself as a $\mathrm{CH}$ based on its remaining energy and mobility. Also, the $\mathrm{MBC}$ protocol considers the connection time in the construction process of clusters to build more reliable path depending on the stability or availability of each link between MN node and $\mathrm{CH}$ node. However in the steady-state phase, data of the sensor nodes are transferred to sink via intracluster and intercluster communication in each round. MBC provides a better performance in mobility-based environment, but it fails to address the critical node occurrence problem, which causes link breakage, packet dropping, and reducing of the network utilization.

5.1.6. Energy-Efficient Competitive Clustering Algorithm. An Energy-Efficient Competitive Clustering (EECC) algorithm using a controlled mobile sink was adopted in [32] to improve the performance of MWSNs and mitigate the hotspot problem. EECC is a distributed clustering algorithm and operates in rounds manner. EECC divides the network into clusters and assigns a $\mathrm{CH}$ for each cluster based on the residual energy of the sensor nodes. Each $\mathrm{CH}$ collects and aggregates data of its members and then waits for arrival of the sink. The mobile sink moves at a certain speed along a predefined line path, which is located at the center of the sensor field, and sojourns at some certain equidistant locations to collect data packets from neighboring $\mathrm{CHs}$ as shown in Figure 5. Simulation results validated that the EECC algorithm with the mobile sink outperforms LEACH [24] in terms of energy utilization and network lifetime.

5.1.7. Mobile Sink Improved Energy-Efficient PEGASIS-Based Routing Protocol. A Mobile sink Improved Energy-Efficient PEGASIS-Based routing protocol (MIEEPB) was presented in [33] to achieve proficient energy utilization of WSNs. MIEEPB divides the sensor field into four regions and constructs a smaller chain in each region. Leader of each chain is selected based on the residual energy and distance from BS that lead to achieving smaller chains and decreasing load of the leader nodes. Then the mobile sink moves with a certain speed along its path and stays for a time at fixed location in each region to guarantee the data collection.

5.1.8. Enhanced Cluster-Based Routing Protocol. Enhanced cluster-based routing protocol has been introduced especially for the MWSN (ECBR-MWSN) [34]. ECBR-MWSN is based on the same principles of its predecessors CBRMobile and LEACH-Mobile. ECBR-MWSN mainly consists of five phases. They are initialization phase, cluster formation phase, CHs selection phase, data transmission phase, and reclustering and rerouting phase. It is aimed at enhancing the lifetime of MWSNs by balancing the consumption energy of the sensor nodes. It selects sensor nodes with highest remaining energy, lowest mobility factor, and least distance from a sink to be $\mathrm{CHs}$. Simulation results clearly showed that the ECBR-MWSN clustering approach is more energy-efficient and hence effective in prolonging the network lifetime compared to LEACH-Mobile and LEACH-ME. 

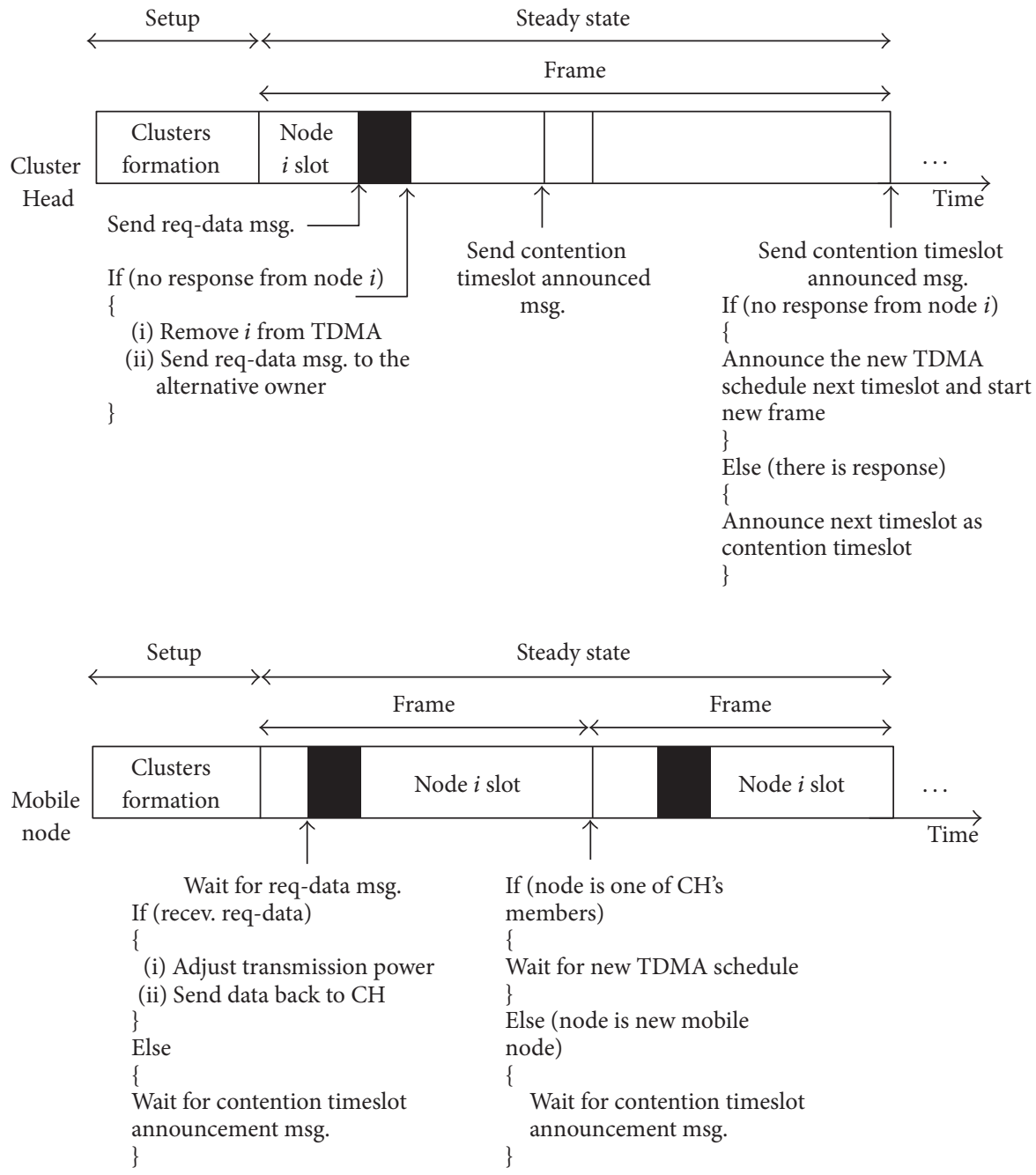

FIGURE 4: Cluster-based routing protocol.

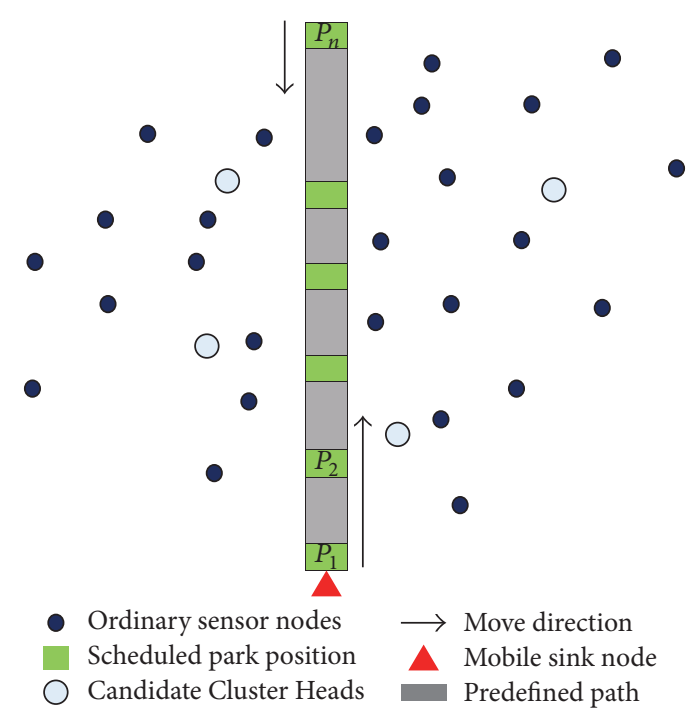

FIGURE 5: EECC algorithm.
5.1.9. Mobility Adaptive Cross-Layer Routing Protocol. In [35] a Mobility Adaptive Cross-layer Routing (MACRO) protocol was designed specifically for the MWSNs. The principle of MACRO is based on a new cross-layer interaction approach among the five reference layers (i.e., application, transport, network, MAC, and physical layers). MACRO tries to sustain the reliability of a route by adapting possible topology changes and channel conditions such as node failures and serious congestion. It uses the route discovery method to reduce the amount of flooding by restricting the subset of nodes able to forward the requests. Additionally, it chooses the most reliable routes for forwarding packets based on the link quality and the mobility of nodes. However, discovering a route causes a significant delay due to the higher number of nodes and the frequent topology change of MWSN. Simulation results showed that the MACRO provides significant improvements on the packet delivery ratio and end-to-end packet delay performances compared to those of the classical CBR-Mobile and LEACH-Mobile routing protocols. 


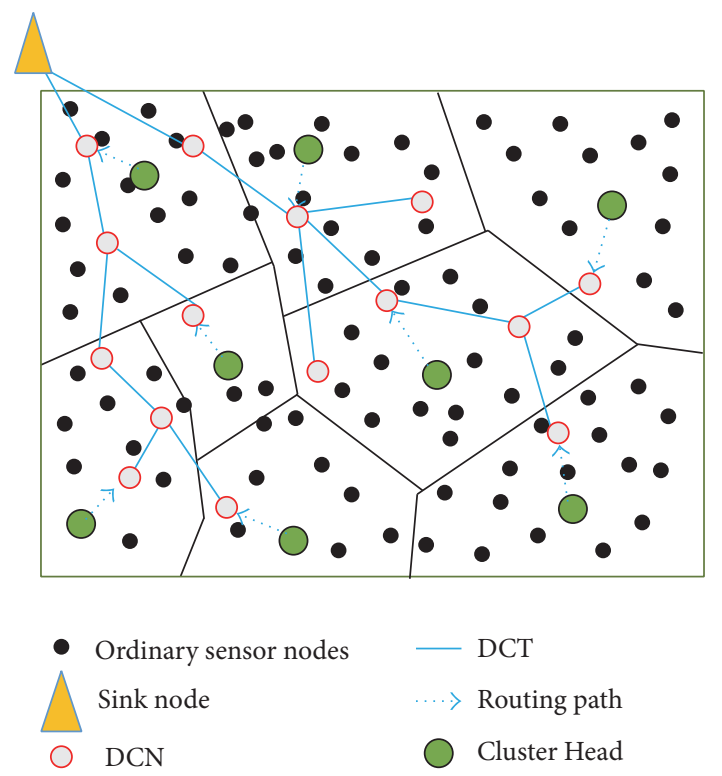

FIgURE 6: CIDT protocol.

5.1.10. Cluster Independent Data Collection Tree Protocol. A Cluster Independent Data collection Tree (CIDT) was designed in [36] based on construction of Data Collection Tree (DCT) to provide a guaranteed reliable network about mobility, traffic, and end-to-end delay. The CIDT consists of a setup phase and a steady-state phase. In the setup phase, cluster formation and tree construction are initiated to identify the optimal path between the members of cluster and sink. In the cluster formation, the selection of $\mathrm{CH}$ and Data Collection Node (DCN) over the network is based on the nodes' residual energy, mobility, Received Signal Strength (RSS), connection time, and cluster dimension. Then DCT is constructed based on the location of selected $\mathrm{CH}$ and the connection time between $\mathrm{CH}$ and DCN as shown in Figure 6. However in the steady-state phase, each $\mathrm{CH}$ collects data from its member nodes and forwards the data to DCN after aggregation. Then DCN again aggregates the data packet from its $\mathrm{CH}$ and forwards it to the sink via the DCT. The simulation results proved that the CIDT protocol provides more stable links, high throughput, good packet delivery ratio with a reduction of the network traffic, and a condensed sum of the energy utilization than LEACH-Mobile and MBC.

5.1.11. Velocity Energy-Efficient and Link-Aware Cluster Tree Protocol. An enhanced version of CIDT protocol, called a Velocity Energy-efficient and Link-aware Cluster Tree (VELCT) scheme, was suggested in [37] for data collection of the MWSNs. VELCT mitigates the existing issues in the CIDT such as coverage, mobility, traffic, tree intensity, and delay of the tree structure. The VELCT scheme consists of a setup phase and a steady-state phase. In the setup phase, clusters are constructed based on the connection time, RSS, coverage time, and robustness for connection. Moreover, the VELCT protocol constructs a DCT between the selected DCN nodes based on the locations of CHs. In the steady-state phase, all $\mathrm{CHs}$ start to collect and aggregate the data from their MNs and then forward the aggregated data to the sink via the DCN nodes. The best construction of DCT enables the VELCT scheme to minimize the energy exploitation, the end-to-end delay, and the traffic of CHs in MWSNs.

5.1.12. Proactive Highly Ambulatory Sensor Routing Protocol. A novel multihop routing protocol for the MWSNs, called Proactive Highly Ambulatory Sensor Routing (PHASeR), was developed in [38] to enable the dynamic and robust routing of data towards the sink. This protocol was motived by radiation mapping applications, which requires the reliable and periodical delivery of measurements to the sink. PHASeR uses a global TDMA-MAC layer to maintain a gradient metric, even in high-speed scenarios. PHASeR does not require any dynamic scheduling because it uses a global TDMA that assigns fixed timeslots for nodes. Also, it uses the blind forwarding technique to pass messages through the network in a multipath manner. The analysis and simulation proved that the PHASeR is effective in a wide array of situations, over varying mobility, scalability, and traffic levels.

5.1.13. Optimizing LEACH Clustering Algorithm. A new clustering algorithm based on combining the use of the LEACH algorithm, mobile sink, and rendezvous points was developed in [39] to reduce the consumption energy. The developed algorithm was called optimizing LEACH. The operation of optimizing LEACH was divided into rounds, where each round starts with a setup phase and follows with a steadystate phase. The setup phase consists of three stages: task ordination, cluster setup, and scheduling. In the task ordination, $\mathrm{CH}$ s and rendezvous nodes (RNs) are selected. Then the clusters are developed based on the distance between sensor nodes and $\mathrm{CH}$ in the cluster setup stage. In the scheduling stage, each $\mathrm{CH}$ broadcasts a schedule to its MNs to organize node transmission timing, while in the steady-state phase, $\mathrm{CHs}$ collect data from their MNs and forward it to the mobile sink or to the nearest living RN. The mobile sink moves along a predetermined line trajectory and sends signals called beacons to notify the RNs about its arrival as shown in Figure 7. Simulation results illustrated that the optimizing LEACH decreases the energy consumption in MWSNs as compared to the traditional LEACH, particularly when the network is large.

5.1.14. Ring Routing Protocol. A ring routing protocol [40] was designed based on a virtual ring structure to mitigate the hotspot problem and minimize the packet delay of large-scale MWSNs. The ring protocol constructs a closed ring loop of a single-node-width depending on the location information of the sensor nodes to advertise the position of the mobile sink with minimal overheads as shown in Figure 8. As the sink moves, it selects anchor nodes (ANs) among its neighbors using the progressive footprint chaining mechanism to relay data of the sensor nodes to the sink. Initially, sink selects the closest node as $\mathrm{AN}$ and broadcasts the location of the selected AN. Then, it selects a new AN and informs the old AN by the position and the MAC address of the new AN, before it leaves the communication range of the old AN. Once the sensor node receives the location of $\mathrm{AN}$, it sends 


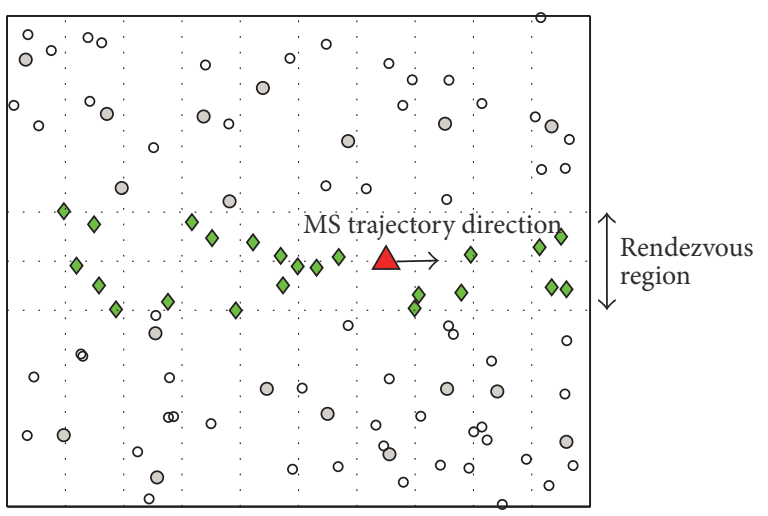

$$
\begin{array}{lll}
\circ \text { Sensor node } & \diamond \mathrm{RN} \\
\circ \mathrm{CH} & \triangle \text { Mobile sink }
\end{array}
$$

FIGURE 7: Optimized LEACH clustering protocol.

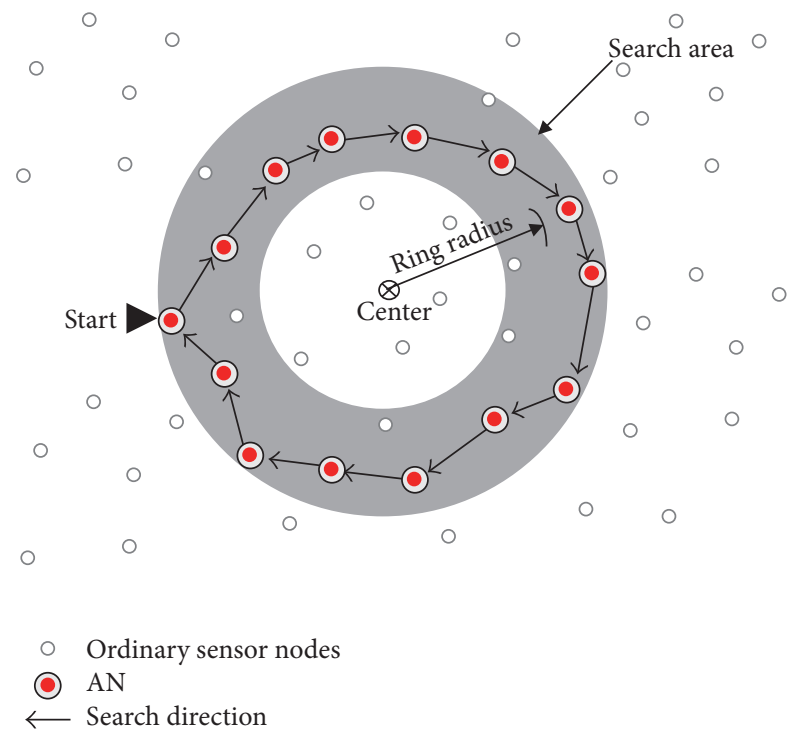

FIGURE 8: Ring routing protocol.

its data directly to this AN. This protocol balances the load among the sensor nodes and solves the hotspot problem by changing the ring structure and switching the role of ring nodes with regular nodes. The simulation results proved that the ring routing is an energy-efficient protocol and improves the network lifetime and the packet delay.

5.1.15. Energy Management Algorithm with Multiple Sinks. An Energy Management algorithm with Multiple Sinks (EMMS) [41] was presented to balance the energy consumption among the sensor nodes. The operation of EMMS is divided into sink moving control and energy management. The sink moving control consists of two steps. In the first step, a closed tour for each mobile sink is determined such that the lengths of the closed tours are roughly equal to balance the workload among the different sinks, while in the second step, the sojourn locations on the closed tour of each mobile sink are determined. The energy management operates into rounds like LEACH. Each round consists of a routing tree construction phase, a sojourn time-calculation phase, and a data collection phase. In the routing tree construction phase, a routing tree at each sojourn location on the closed tour of each mobile sink is constructed based on the residual energy of the sensor nodes, while in the sojourn time-calculation phase, the sojourn time at each sojourn location on the closed tour of each sink is calculated based on the members of the constructed tree. The data of the sensor nodes are transferred to the mobile sink via the constructed tree in the data collection phase. At the end of data transmission, the mobile sink collects the residual energy information from nodes in the neighbor set and then travels to its next sojourn location. Simulation results demonstrated that the EMMS algorithm improves the energy-efficiency and the data transmission quantity within the network significantly.

5.1.16. Anycast Tree-Based Routing Protocol. An anycast treebased routing protocol [42] was developed for WSNs with mobile sensors and mobile multiple sinks to reduce the network traffic and the energy consumption. The operation of this developed protocol is based on expanding ring search, anycast messaging, and reactive mode with maintaining the route state information in the sensor nodes. The developing protocol constructs and maintains a routing tree among the sensor nodes and sink. For sending data from a sensor node to the sink via the established route, all sensor nodes on this route use the unicast mode of request transmission instead of the broadcast mode. The developed protocol does not require any control messages because each node keeps only address of the next node on its path to the sink.

5.2. Optimized Hierarchical-Based Routing Protocols. The objectives of hierarchal routing protocols are saving the dissipated energy, ensuring the network connectivity, and prolonging the lifetime of the MWSNs. These objectives can be achieved via finding the optimal head nodes and controlling the mobility of the mobile elements in the MWSNs. This is a difficult problem and can be considered as a Nondeterministic Polynomial (NP) optimization problem. In order to solve and find optimal solutions for this problem, researchers have developed some robust hierarchical routing protocols based on the optimization algorithms, such as Particle Swarm Optimization (PSO), Fuzzy Logic (FL), Genetic Algorithm (GA), and Artificial Bee Colony (ABC). In this subsection, we review some of the recently optimized hierarchical-based routing protocols that are developed for the MWSNs.

5.2.1. PSO-Based Routing Protocol with Mobile Base Station. An energy-efficient routing protocol based on PSO with mobile base station (PSO-MBS) was presented in [43] to improve the lifetime and the data delivery of WSNs. The aim of PSO-MBS is finding the sojourn positions of the mobile BS and partitioning the network into clusters for gathering data of the sensor nodes. The operation of the PSO-MBS protocol begins with the setup phase followed by rounds of the steady-state phase. In the setup phase, BS uses the PSO to find the optimal sojourn positions of BS based on the locations of the sensor nodes and the number of clusters, 


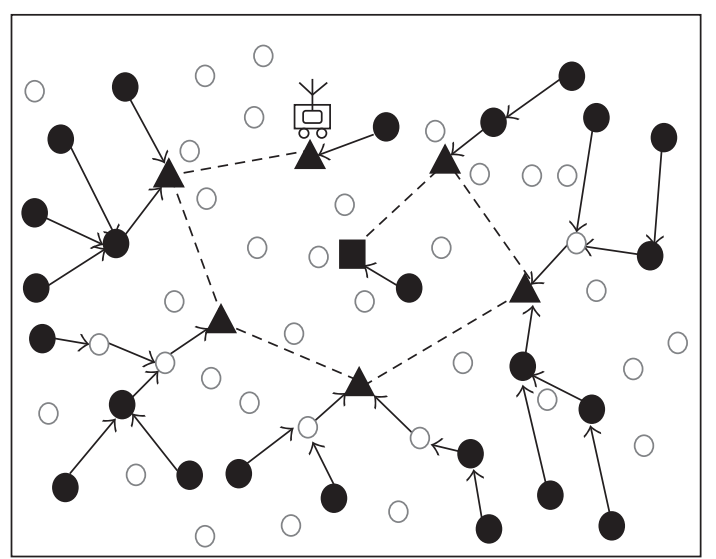

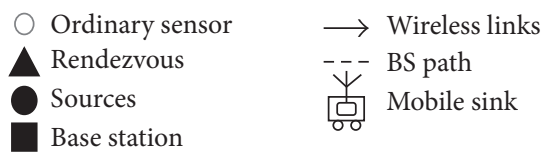

FIGURE 9: Rendezvous routing algorithm.

while in the steady-state phase, each sensor node sends its data to its $\mathrm{CH}$ using the TDMA schedule. Then $\mathrm{CH}$ aggregates the received data and forwards it to BS when BS visits its cluster. Simulation results clearly showed that the PSO-MBS outperforms the other protocols in terms of the network lifetime and the data delivery.

5.2.2. Genetic Algorithm Based Routing Protocol. A Genetic Algorithm based routing protocol called GAROUTE [44] was presented to build a stable clustering network. GAROUTE was designed to work with mobile sensor nodes, which are not aware of their locations. GAROUTE is a centralized algorithm, in which BS broadcasts a request message to all nodes on the network asking each node to send a list of its neighboring nodes within its transmission range. Each node finds its list of neighbors by broadcasting a message to notify these neighbors about its presence. Then a node directly sends this list along with its own speed and energy information to BS. GAROUTE uses the obtained information to construct stable clusters with best CHs using the Genetic Algorithm based on minimizing the mean communication energy, $\mathrm{CH}$ fraction, and total $\mathrm{CH}$ speed.

5.2.3. Efficient Rendezvous Routing Algorithms. Efficient rendezvous routing algorithms [45] were developed to improve the performance of MWSNs using mobile base stations (BSs). The first rendezvous algorithm finds a set of sensor nodes to act as Rendezvous Points (RPs) based on minimizing the transmission cost. The determined RPs collect and aggregate data from the sensor nodes and then transfer it to the mobile BS when it arrives as shown in Figure 9. The first algorithm assumes that the BS moves freely to collect data from RPs, while the second algorithm was designed for BSs that move along fixed paths. The second algorithm is executed by BS to find the data collection tour, the set of sensor nodes that act as RPs on the tour, and the routing trees among the sensor nodes that are rooted at RPs based on minimizing the total edge length of the constructed trees. Simulation results illustrated that the developed algorithms can achieve satisfactory performance under a range of settings.

5.2.4. PSO-Based Clustering Protocol for Heterogeneous Network. A new clustering protocol based on PSO [46] was designed to improve the lifetime and avoid the energy hole problem of the heterogeneous WSNs. The developed protocol considers a mobile sink and adaptive transmission power control to improve the coverage and balance the network load. Also, the PSO is used to find the optimal and the shortest path between $\mathrm{CH}$ s through which the mobile sink travels. Due to changing the neighbors of the sink in each round, the developed protocol mitigates the energy hole problem and hence the network lifetime is increased.

5.2.5. Clustering Algorithm Based on Glowworm Swarm Optimization. A Clustering Algorithm based on Glowworm swarm optimization with Mobile sink (CAGM) [47] was developed to balance the energy consumption and maximize the lifetime of WSNs. CAGM protocol utilizes the glowworm swarm optimization algorithm to divide the network into several clusters and select the best $\mathrm{CH}$ for each cluster based on minimizing the consumption energy. Each $\mathrm{CH}$ collects data from its MNs and aggregates it before forwarding it to a mobile sink. CAGM uses a mobile sink to balance the energy consumption among the sensor nodes. The mobile sink roams around the network to collect data from $\mathrm{CHs}$ through a really short communication range. Simulation results showed that the CAGM protocol could efficiently prolong the lifetime of WSNs.

5.2.6. Multiobjective Evolutionary Routing Protocol. A NonDominated Sorting Genetic Algorithm-II based Routing Protocol (NSGAII-RP) was presented in [48] to build an efficient routing and increase the lifetime of the MWSNs. NSGAII$\mathrm{RP}$ protocol considers the coverage and the routing problem. NSGAII-RP optimizes the coordinates of the mobile nodes and finds the optimal CHs using the NSGAII algorithm based on minimizing the energy consumption and ensuring the network coverage. Simulation results clearly showed that NSGAII-RP could build energy-efficient routing and ensure the network coverage; as a result the network lifetime is maximized.

5.2.7. Optimized Zone-Based Energy-Efficient Routing Protocol. An Optimized Zone-based Energy-Efficient Routing Protocol (OZEEP) [49] was adopted to improve the lifetime of MWSNs. OZEEP optimizes the clustering and the $\mathrm{CH}$ selection process using the Genetic Fuzzy System (GFS) in two steps. The first step is the screening process, in which all nodes use the fuzzy system to elect some nodes for the role of $\mathrm{CH}$ based on their residual energy, distance from BS, neighbors, and mobility. The elected nodes declare themselves as candidates for $\mathrm{CH}$ to BS. However in the second step, BS runs the Genetic Algorithm based on the received information from the candidate nodes and makes a final selection of the nodes to take up the $\mathrm{CH}$ role based on the 


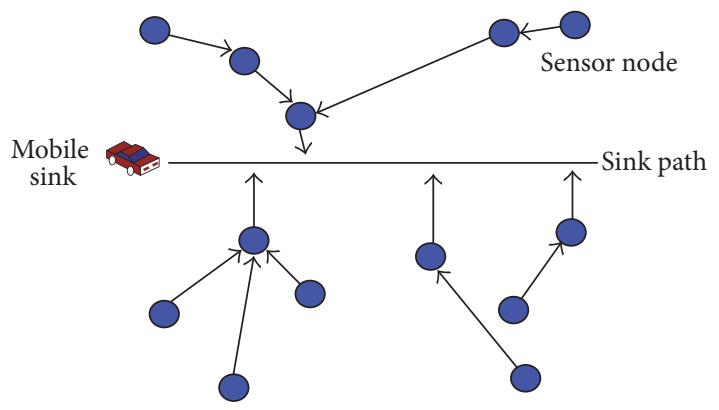

FIGURE 10: MLS based routing protocol.

consumption energy, mobility factor, and the number of $\mathrm{CHs}$. Using the fuzzy system in the first step eases the task of the genetic system. Simulation results clearly showed that the OZEEP improves the network lifetime and the packet delivery ratio.

5.2.8. Lifetime Optimization Algorithm with Mobile Sink Nodes. A Lifetime Optimization Algorithm with Mobile Sink Nodes (LOA-MSN) [50] was designed to overcome the energy hole problem in WSNs. LOA-MSN uses a hybrid positioning algorithm of satellite positioning and RSS positioning to obtain locations of all sensor nodes in the network with minimum energy consumption. LOA-MSN divides the sensor field into several grids of the same size and each grid is numbered. The LOA-MSN considers the constraints of the movement path constraints, flow, energy consumption, and the link transmission in the clustering problem. This problem is divided into movement path selection model and lifetime optimization model with known movement paths. These models are solved using the distributed asynchronous Bellman-Ford algorithm. Simulation results proved that LOA-MSN makes full use of the node's energy to prolong the network lifetime.

5.2.9. Energy-Efficient Routing Based on Minimum Load Set Algorithm. An energy-efficient routing based on Minimum Load Set (MLS) algorithm [51] was developed for prolonging the lifetime of the large-scale WSNs using a mobile sink. MLS utilizes the mobile sink to gather data periodically along the predefined path and each sensor node uploads its data to the mobile sink over a multihop communication path as shown in Figure 10. MLS constructs a routing tree by growing from the fictitious root node and adds one node to the tree at each iteration by avoiding burdening the nodes that already have heavier loads. The MLS algorithm operates in an iteration manner, where each iteration consists of the primary selection step and the final selection step. In the first step, MLS selects the most appropriate candidate edge for each visited node from its adjacent edges, while in the final selection step, MLS determines the candidate edge that has the optimal expectant load set among the candidate edges of all visited nodes by primary selection and adds the candidate edge to the growing tree.

5.2.10. Artificial Bee Colony Based Data Collection Mechanism. Yue et al. [52] designed an efficient and reliable data collection mechanism for MWSNs based on the Artificial Bee Colony (ABC). The designed protocol utilizes a mobile sink to collect data of the sensor nodes. Also, it uses the ABC algorithm to find the reliable routing and the movement path of the mobile sink. The operation of the designed protocol consists of an initial phase and a data collection phase in each round. In the initial phase, the mobile sink uses the network topology information to construct optimal clusters with the best $\mathrm{CH}$ nodes and build a routing tree among the selected $\mathrm{CHs}$, while in the data collection phase, each $\mathrm{CH}$ collects the sensory information of its member nodes before the arrival of the mobile sink. Once the sink receives the collected data, it calculates and chooses the next hop's objective position according to the current network environment parameters and the mobile strategy using the $\mathrm{ABC}$ algorithm to obtain the shortest route to each $\mathrm{CH}$ and node as shown in Figure 11. Simulation results showed that, compared to similar existing algorithms, the designed protocol improves the network throughput, collects data more efficiently, and saves energy.

\section{Classification and Comparison}

A detailed classification of the reviewed hierarchical routing protocols based on different criteria is given in Table 1. Table 2 shows a comparison among these protocols based on delay, network size, energy-efficiency, and scalability while highlighting the advantages and disadvantages of each protocol.

\section{Conclusion}

Recently, many routing protocols are developed especially to support mobility of Wireless Sensor Networks. This paper reviews the recently hierarchical-based routing protocols that are developed in the last five years for the Mobile Wireless Sensor Networks (MWSNs). In this survey, the hierarchicalbased routing protocols are grouped into classical-based routing and optimized-based routing. Also, a detailed classification of the reviewed protocols based on different metrics, such as control manner, network architecture, mobile element, mobility pattern, clustering attributes, protocol operation, path establishment, communication paradigm, energy model, protocol objectives, and applications, is presented in this paper. Moreover, the reviewed protocols are evaluated and compared on the basis of delay, network size, and energyefficiency while highlighting the features and drawbacks of each protocol. This survey can be helpful for designers of MWSNs in selection of an appropriate hierarchical routing protocol for a specific application that requires mobile nodes.

The effort in this area should be continued in the area of the hierarchical routing to improve the performance of MWSNs. Two open issues should be considered in the future research. First issue is the real-work implantations. Simulation of the routing protocols depends on modeling the hardware of the sensor nodes. However, the mathematic models do not exactly simulate the real world. Simulation should be just the first step towards hardware implementations. Experiments with real testbeds force the experimenter 


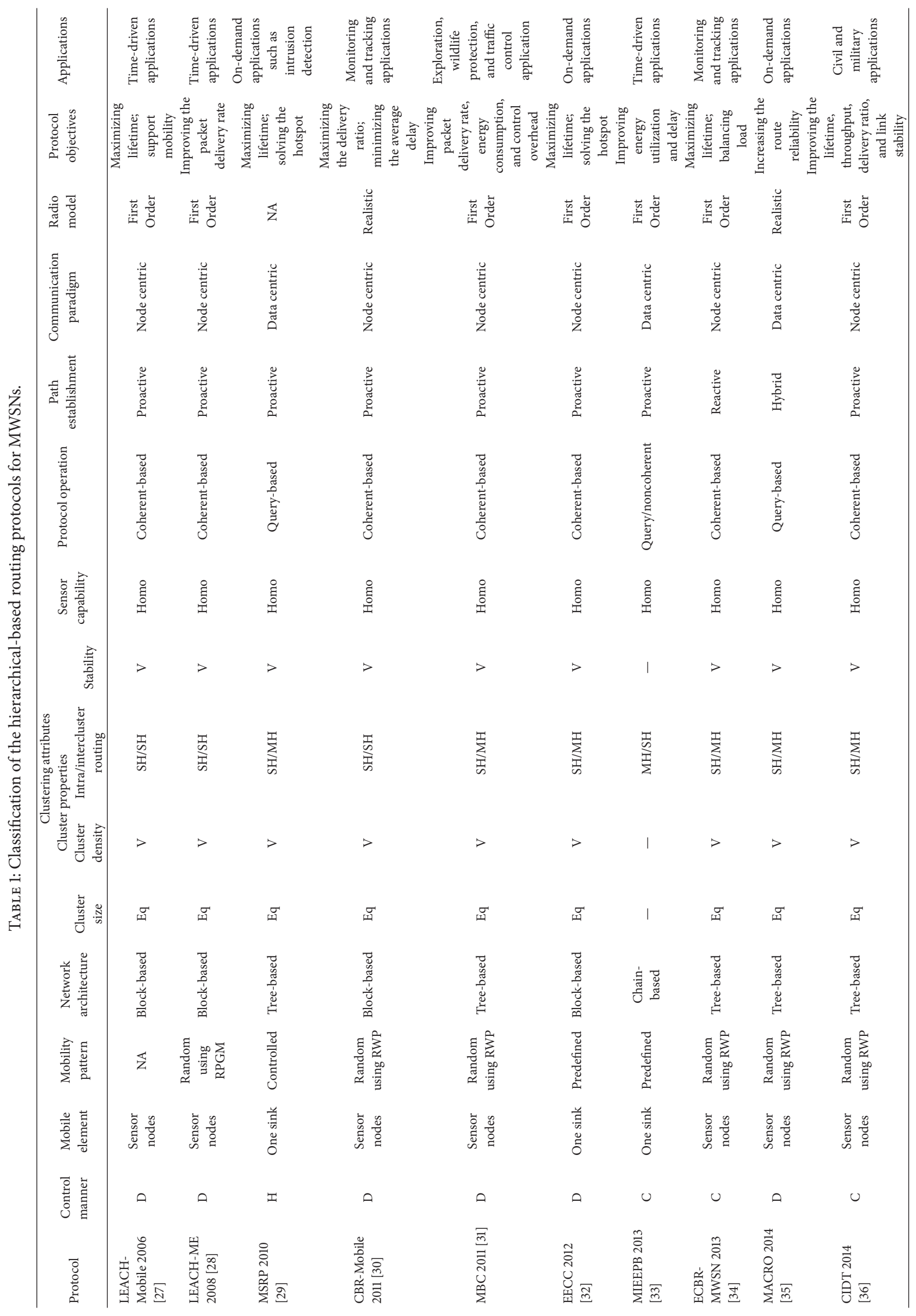




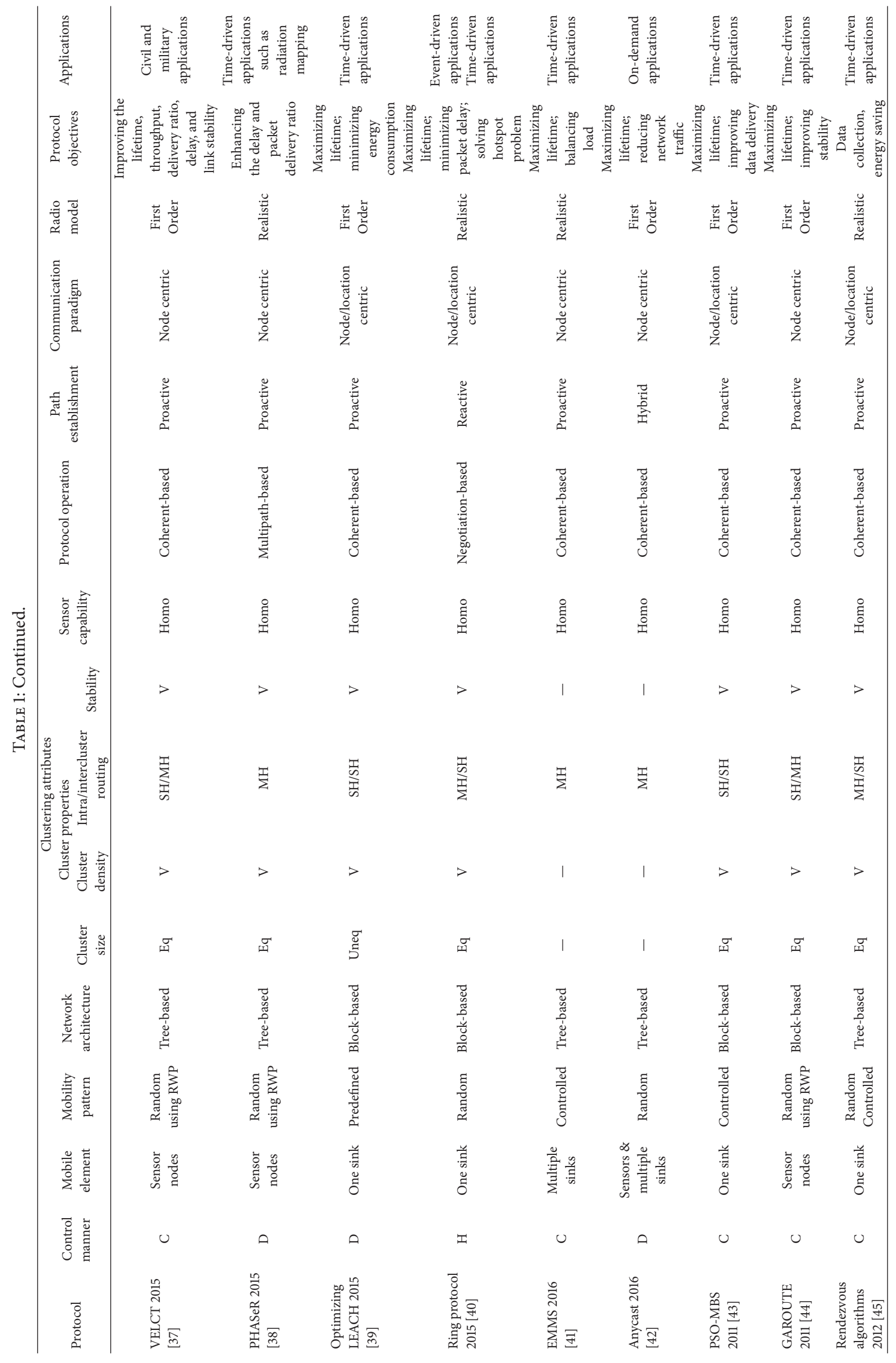




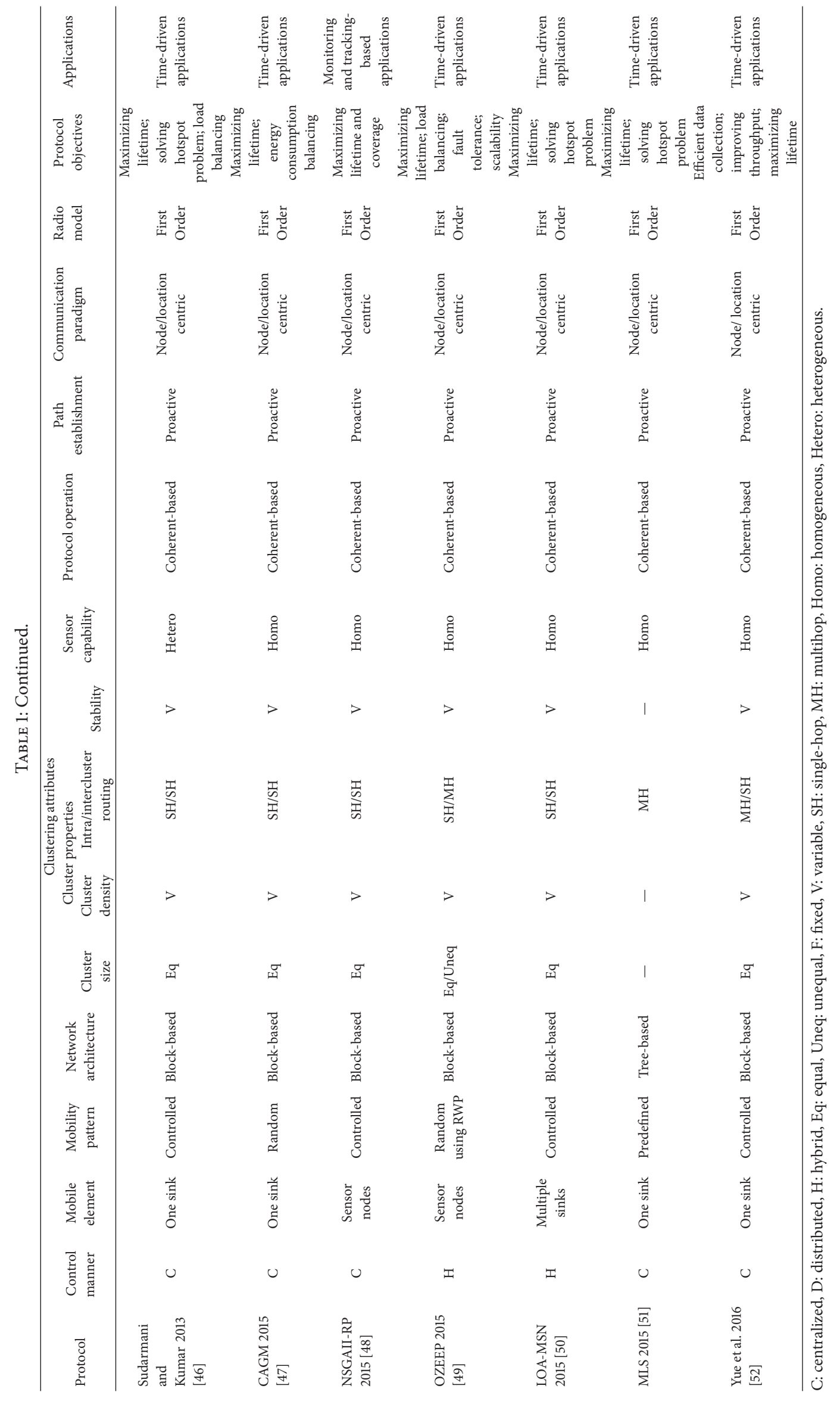




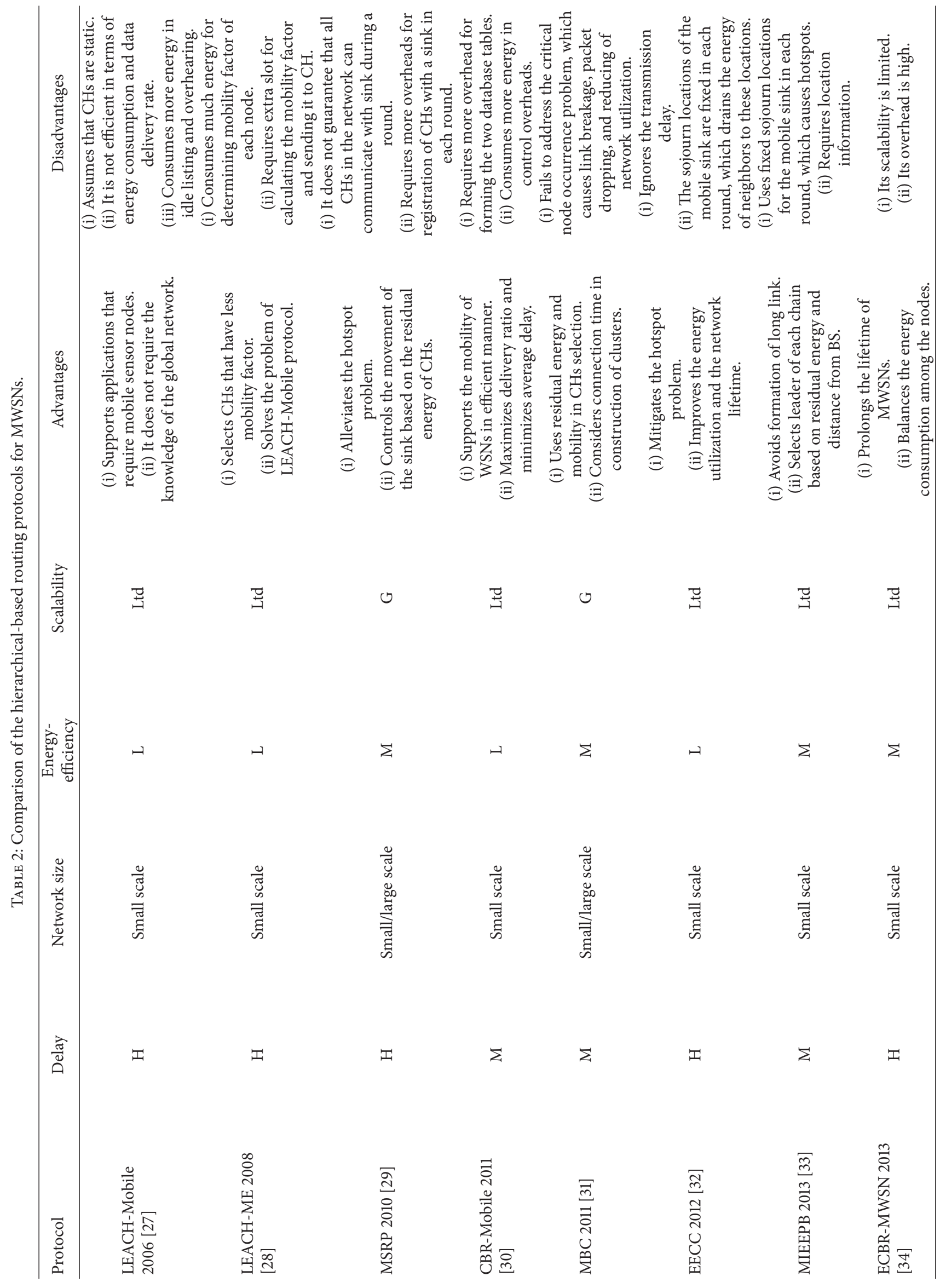




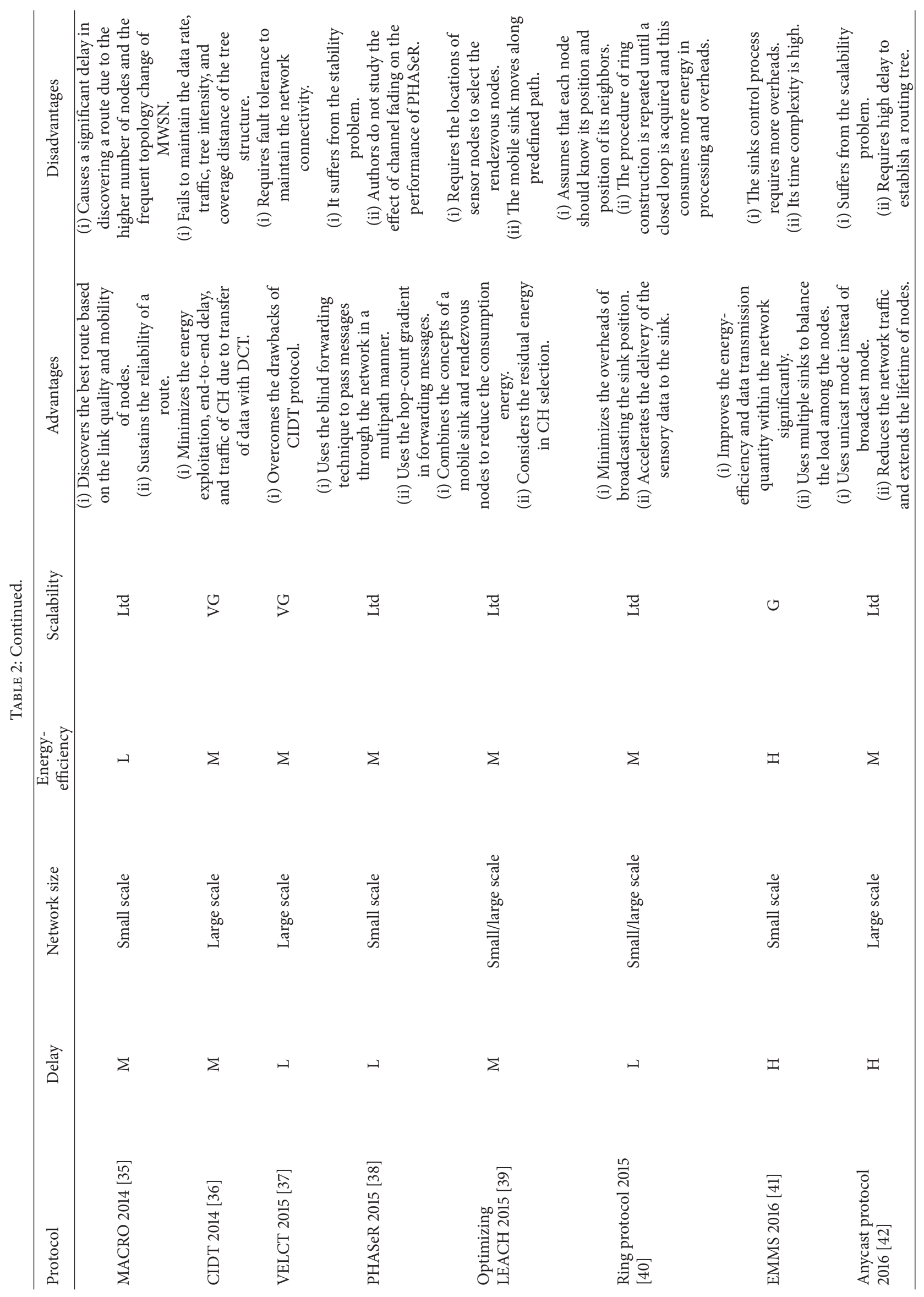




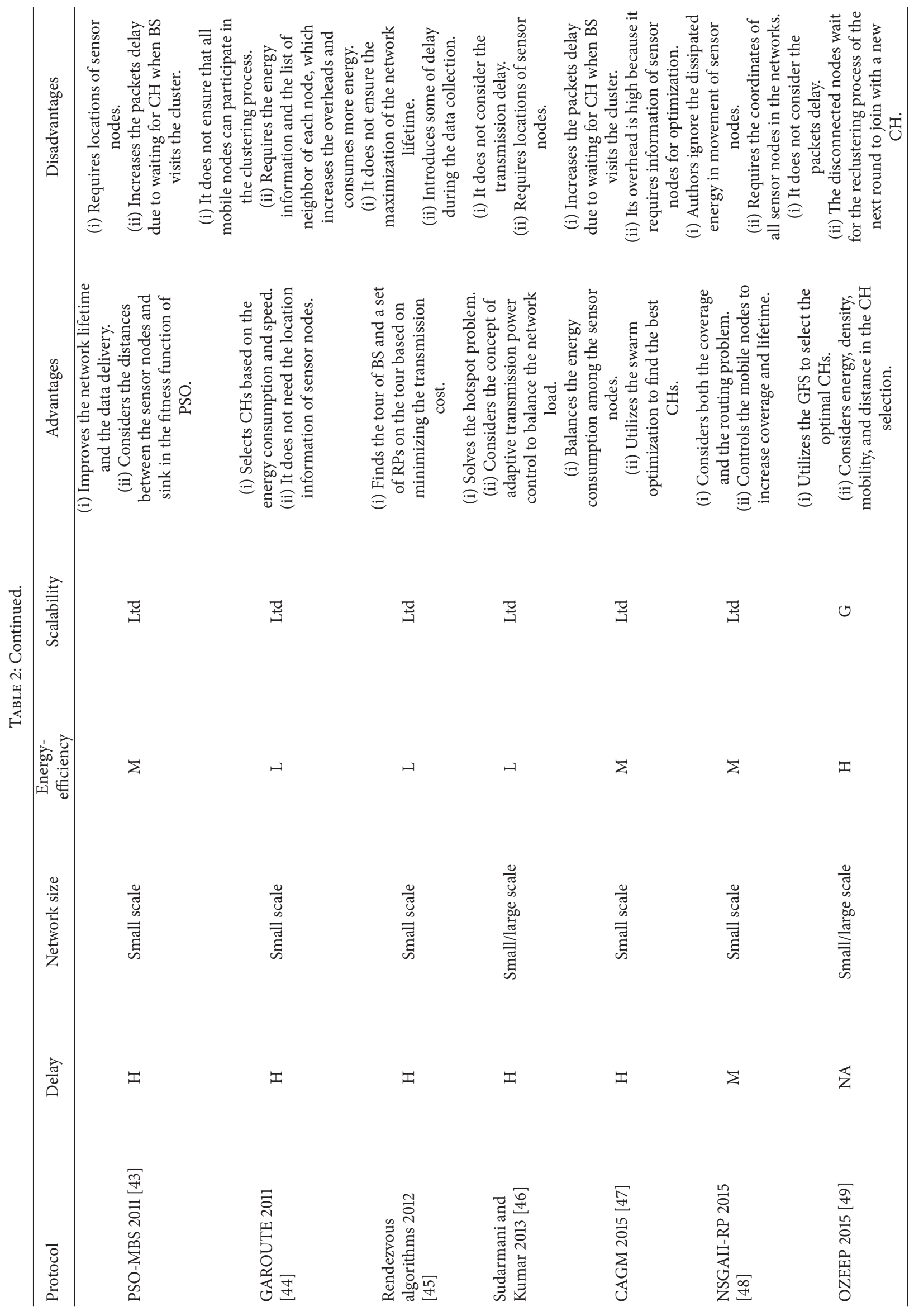




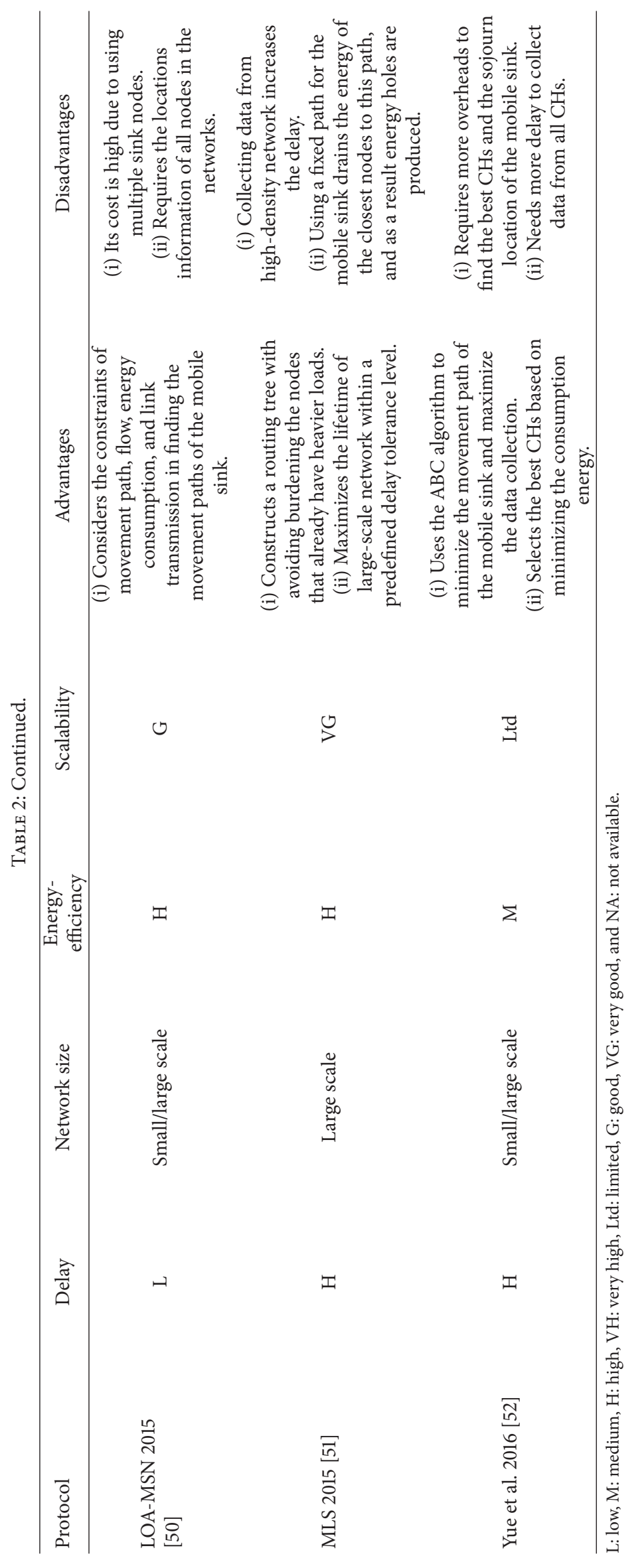




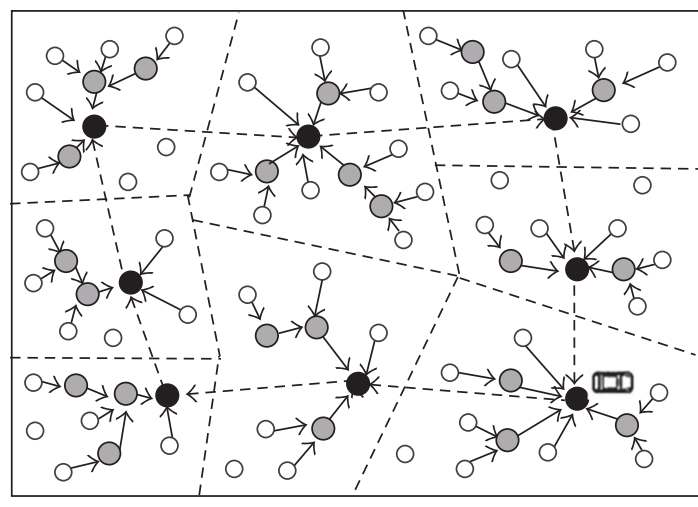

$\begin{array}{llll}\odot & \text { Sensor node } & \leftarrow-\text { Sink path } \\ \longleftarrow & \text { Routing path } & \text { Cluster node } \\ & \text { Route node } & \text { Mobile sink }\end{array}$

FIgURE 11: ABC-based data collection mechanism.

to face a wide set of problems and challenges that can hardly be replicated in simulation. Second issue is the overheads and computational time of clustering the network. A centralized routing is more energy-efficient than the distributed routing. However in the centralized routing, all sensor nodes should send their information such as their locations and energy to BS. This takes some time and increases the overheads of protocol especially in the dense networks and the BS is far away from the sensor field. Designers should develop semidistributed or semicentralized protocols that run within Cluster Head rather than sink.

\section{Competing Interests}

The authors declare that they have no competing interests.

\section{Acknowledgments}

The authors would like to acknowledge the Egyptian Ministry of Higher Education and the Egyptian Cultural and Educational Bureau in Tokyo for funding the Ph.D. studies of Nabil Sabor at Niigata University, Electrical and Electronic Engineering Department.

\section{References}

[1] S. Chellappan, X. Bai, B. Ma, D. Xuan, and C. Xu, "Mobility limited flip-based sensor networks deployment," IEEE Transactions on Parallel and Distributed Systems, vol. 18, no. 2, pp. 199-211, 2007.

[2] K. Dantu, M. Rahimi, H. Shah, S. Babel, A. Dhariwal, and G. S. Sukhatme, "Robomote: enabling mobility in sensor networks," in Proceedings of the 4th International Symposium on Information Processing in Sensor Networks (IPSN '05), pp. 404-409, Boise, Idaho, USA, April 2005.

[3] O. Gaddour, A. Koubaa, R. Rangarajan, O. Cheikhrouhou, E. Tovar, and M. Abid, "Co-RPL: RPL routing for mobile low power wireless sensor networks using Corona mechanism," in Proceedings of the 9th IEEE International Symposium on
Industrial Embedded Systems (SIES '14), pp. 200-209, IEEE, Pisa, Italy, June 2014.

[4] X. Lai, Q. Liu, X. Wei, W. Wang, G. Zhou, and G. Han, "A survey of body sensor networks," Sensors, vol. 13, no. 5, pp. 5406-5447, 2013.

[5] S. B. Awwad, C. Ng, N. Noordin, M. F. Rasid, and A. R. H. Alhawari, "Mobility and traffic adapted Cluster Based Routing for Mobile Nodes (CBR-Mobile) protocol in wireless sensor networks," in Ad Hoc Networks: Second International Conference, ADHOCNETS 2010, Victoria, BC, Canada, August 18-20, 2010, Revised Selected Papers, vol. 49 of Lecture Notes of the Institute for Computer Sciences, Social Informatics and Telecommunications Engineering, pp. 281-296, Springer, Berlin, Germany, 2010.

[6] R. Silva, Z. Zinonos, J. S. Silva, and V. Vassiliou, "Mobility in WSNs for critical applications," in Proceedings of the 16th IEEE Symposium on Computers and Communications (ISCC '11), pp. 451-456, Corfu, Greece, June 2011.

[7] I. F. Akyildiz, W. Su, Y. Sankarasubramaniam, and E. Cayirci, "A survey on sensor networks," IEEE Communications Magazine, vol. 40, no. 8, pp. 102-114, 2002.

[8] T. P. Lambrou and C. G. Panayiotou, "A survey on routing techniques supporting mobility in sensor networks," in Proceedings of the 5th International Conference on Mobile Ad-Hoc and Sensor Networks (MSN'09), pp. 78-85, IEEE, Fujian, China, December 2009.

[9] M. Di Francesco, S. K. Das, and G. Anastasi, "Data collection in wireless sensor networks with mobile elements: a survey," $A C M$ Transactions on Sensor Networks, vol. 8, no. 1, article 7, 2011.

[10] J. Lakhotia and R. Kumar, "Cluster based routing protocols for mobile wireless sensor network: a review," International Journal of Advanced Research in Computer Engineering \& Technology (IJARCET), vol. 3, pp. 2274-2278, 2014.

[11] G. S. Sara and D. Sridharan, "Routing in mobile wireless sensor network: a survey," Telecommunication Systems, vol. 57, no. 1, pp. 51-79, 2014.

[12] J. Wang, X. Yang, Z. Zhang, B. Li, and J.-U. Kim, "A survey about routing protocols with mobile sink for wireless sensor network," International Journal of Future Generation Communication and Networking, vol. 7, no. 5, pp. 221-228, 2014.

[13] S. Yu, B. Zhang, C. Li, and H. T. Mouftah, "Routing protocols for wireless sensor networks with mobile sinks: a survey," IEEE Communications Magazine, vol. 52, no. 7, pp. 150-157, 2014.

[14] C. Tunca, S. Isik, M. Y. Donmez, and C. Ersoy, "Distributed mobile sink routing for wireless sensor networks: a survey," IEEE Communications Surveys and Tutorials, vol. 16, no. 2, pp. 877-897, 2014.

[15] Padmavati and T. C. Aseri, "Comparison of routing protocols in wireless sensor network using mobile sink-a survey," in Proceedings of the Recent Advances in Engineering and Computational Sciences (RAECS '14), pp. 1-4, March 2014.

[16] S. R. Jain and N. V. Thakur, "Overview of cluster based routing protocols in static and mobile wireless sensor networks," in Information Systems Design and Intelligent Applications: Proceedings of Second International Conference INDIA 2015, Volume 1, pp. 619-626, Springer India, New Delhi, India, 2015.

[17] T. Camp, J. Boleng, and V. Davies, "A survey of mobility models for ad hoc network research," Wireless Communications and Mobile Computing, vol. 2, no. 5, pp. 483-502, 2002.

[18] R. A. Pushpa, A. Vallimayil, and V. R. S. Dhulipala, "Impact of mobility models on mobile sensor networks," in Proceedings 
of the 3rd International Conference on Electronics Computer Technology (ICECT '11), pp. 102-106, IEEE, Kanyakumari, India, April 2011.

[19] G. Jayakumar and G. Ganapathi, "Reference point group mobility and random waypoint models in performance evaluation of MANET routing protocols," Journal of Computer Systems, Networks, and Communications, vol. 2008, Article ID 860364, 10 pages, 2008.

[20] X. Wu, G. Chen, and S. K. Das, "On the energy hole problem of nonuniform node distribution in wireless sensor networks," in Proceedings of the IEEE International Conference on Mobile Ad Hoc and Sensor Sysetems (MASS '06), pp. 180-187, Vancouver, Canada, October 2006.

[21] X. Liu, "A survey on clustering routing protocols in wireless sensor networks," Sensors, vol. 12, no. 8, pp. 11113-11153, 2012.

[22] S. K. Gupta, N. Jain, and P. Sinha, "Clustering protocols in wireless sensor networks: a survey," International Journal of Applied Information Systems, vol. 5, no. 2, pp. 41-50, 2013.

[23] M. N. Halgamuge, M. Zukerman, K. Ramamohanarao, and H. L. Vu, "An estimation of sensor energy consumption," Progress in Electromagnetics Research B, vol. 12, pp. 259-295, 2009.

[24] W. R. Heinzelman, A. Chandrakasan, and H. Balakrishnan, "Energy-efficient communication protocol for wireless microsensor networks," in Proceedings of the 33rd Annual Hawaii International Conference on System Sciences (HICSS '00), pp. 1-10, Maui, Hawaii, USA, January 2000.

[25] "CC2420: Single-Chip 2.4 GHz IEEE 802.15.4 Compliant and ZigBee $^{\mathrm{TM}}$ Ready RF Transceiver," http://www.ti.com/product/ cc2420.

[26] Y.-M. Huang, M.-Y. Hsieh, and F. Sandnes, "Wireless sensor networks and applications," in Sensors, S. C. Mukhopadhyay and R. Y. M. Huang, Eds., vol. 21, pp. 199-219, Springer, Berlin, Germany, 2008.

[27] K. Do-Seong and C. Yeong-Jee, "Self-organization routing protocol supporting mobile nodes for wireless sensor network," in Proceedings of the 1st International Multi-Symposiums on Computer and Computational Sciences (IMSCCS '06), pp. 622626, Hangzhou, China, June 2006.

[28] G. S. Kumar, M. V. Vinu Paul, G. Athithan, and K. P. Jacob, "Routing protocol enhancement for handling node mobility in wireless sensor networks," in Proceedings of the IEEE Region 10 Conference (TENCON '08), pp. 1-6, IEEE, Hyderabad, India, November 2008.

[29] B. Nazir and H. Hasbullah, "Mobile Sink based Routing Protocol (MSRP) for prolonging network lifetime in clustered wireless sensor network," in Proceedings of the International Conference on Computer Applications and Industrial Electronics (ICCAIE '10), pp. 624-629, Kuala Lumpur, Malaysia, December 2010.

[30] S. A. B. Awwad, C. K. Ng, N. K. Noordin, and M. F. A. Rasid, "Cluster based routing protocol for mobile nodes in wireless sensor network," Wireless Personal Communications, vol. 61, no. 2, pp. 251-281, 2011.

[31] S. Deng, J. Li, and L. Shen, "Mobility-based clustering protocol for wireless sensor networks with mobile nodes," Wireless Sensor Systems, IET, vol. 1, no. 1, pp. 39-47, 2011.

[32] J. Wang, X. Yang, T. Ma, M. Wu, and J.-U. Kim, "An energyefficient competitive clustering algorithm for wireless sensor networks using mobile sink," International Journal of Grid and Distributed Computing, vol. 5, pp. 79-92, 2012.
[33] M. R. Jafri, N. Javaid, A. Javaid, and Z. A. Khan, "Maximizing the lifetime of multi-chain PEGASIS using sink mobility," World Applied Sciences Journal, vol. 21, no. 9, pp. 1283-1289, 2013.

[34] R. U. Anitha and P. Kamalakkannan, "Enhanced cluster based routing protocol for mobile nodes in wireless sensor network," in Proceedings of the International Conference on Pattern Recognition, Informatics and Mobile Engineering (PRIME '13), pp. 187193, Salem, India, February 2013.

[35] S. Cakici, I. Erturk, S. Atmaca, and A. Karahan, "A novel crosslayer routing protocol for increasing packet transfer reliability in mobile sensor networks," Wireless Personal Communications, vol. 77, no. 3, pp. 2235-2254, 2014.

[36] R. Velmani and B. Kaarthick, "An energy efficient data gathering in dense mobile wireless sensor networks," ISRN Sensor Networks, vol. 2014, Article ID 518268, 10 pages, 2014.

[37] R. Velmani and B. Kaarthick, "An efficient cluster-tree based data collection scheme for large mobile wireless sensor networks," IEEE Sensors Journal, vol. 15, no. 4, pp. 2377-2390, 2015.

[38] T. Hayes and F. H. Ali, "Proactive Highly Ambulatory Sensor Routing (PHASeR) protocol for mobile wireless sensor networks," Pervasive and Mobile Computing, vol. 21, pp. 47-61, 2015.

[39] S. Mottaghi and M. R. Zahabi, "Optimizing LEACH clustering algorithm with mobile sink and rendezvous nodes," AEUInternational Journal of Electronics and Communications, vol. 69, no. 2, pp. 507-514, 2015.

[40] C. Tunca, S. Isik, M. Y. Donmez, and C. Ersoy, "Ring routing: an energy-efficient routing protocol for wireless sensor networks with a mobile sink," IEEE Transactions on Mobile Computing, vol. 14, no. 9, pp. 1947-1960, 2015.

[41] J. Shi, X. Wei, and W. Zhu, "An efficient algorithm for energy management in wireless sensor networks via employing multiple mobile sinks," International Journal of Distributed Sensor Networks, vol. 2016, Article ID 3179587, 9 pages, 2016.

[42] A. E. Kostin, Y. Fanaeian, and H. Al-Wattar, "Anycast tree-based routing in mobile wireless sensor networks with multiple sinks," Wireless Networks, vol. 22, no. 2, pp. 579-598, 2016.

[43] N. A. A. Latiff, N. M. A. Latiff, and R. B. Ahmad, "Prolonging lifetime of wireless sensor networks with mobile base station using particle swarm optimization," in Proceedings of the 4th International Conference on Modeling, Simulation and Applied Optimization (ICMSAO '11), pp. 1-6, IEEE, Kuala Lumpur, Malaysia, April 2011.

[44] S. Sarangi and S. Kar, "Genetic algorithm based mobility aware clustering for energy efficient routing in wireless sensor networks," in Proceedings of the 17th IEEE International Conference on Networks (ICON '11), pp. 1-6, Singapore, December 2011.

[45] G. Xing, M. Li, T. Wang, W. Jia, and J. Huang, "Efficient rendezvous algorithms for mobility-enabled wireless sensor networks," IEEE Transactions on Mobile Computing, vol. 11, no. 1, pp. 47-60, 2012.

[46] R. Sudarmani and K. R. S. Kumar, "Particle swarm optimization-based routing protocol for clustered heterogeneous sensor networks with mobile sink," American Journal of Applied Sciences, vol. 10, no. 3, pp. 259-269, 2013.

[47] J. Wang, Y.-Q. Cao, B. Li, S.-Y. Lee, and J.-U. Kim, "A glowworm swarm optimization based clustering algorithm with mobile sink support for wireless sensor networks," Journal of Internet Technology, vol. 16, no. 5, pp. 825-832, 2015.

[48] B. A. A. Attea, E. A. Khalil, and A. Cosar, "Multi-objective evolutionary routing protocol for efficient coverage in mobile sensor networks," Soft Computing, vol. 19, no. 10, pp. 2983-2995, 2015. 
[49] J. R. Srivastava and T. S. B. Sudarshan, "A genetic fuzzy system based optimized zone based energy efficient routing protocol for mobile sensor networks (OZEEP)," Applied Soft Computing Journal, vol. 37, pp. 863-886, 2015.

[50] Y. Chen, Z. Wang, T. Ren, and H. Lv, "Lifetime optimization algorithm with mobile sink nodes for wireless sensor networks based on location information," International Journal of Distributed Sensor Networks, vol. 2015, Article ID 857673, 11 pages, 2015.

[51] H. Zhao, S. Guo, X. Wang, and F. Wang, "Energy-efficient topology control algorithm for maximizing network lifetime in wireless sensor networks with mobile sink," Applied Soft Computing, vol. 34, pp. 539-550, 2015.

[52] Y. Yue, J. Li, H. Fan, and Q. Qin, "Optimization-based artificial bee colony algorithm for data collection in large-scale mobile wireless sensor networks," Journal of Sensors, vol. 2016, Article ID 7057490, 12 pages, 2016. 


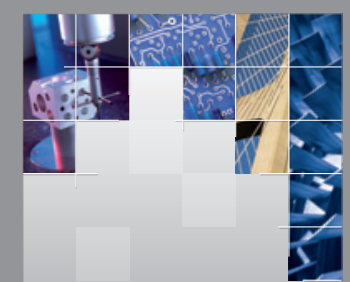

\section{Enfincering}
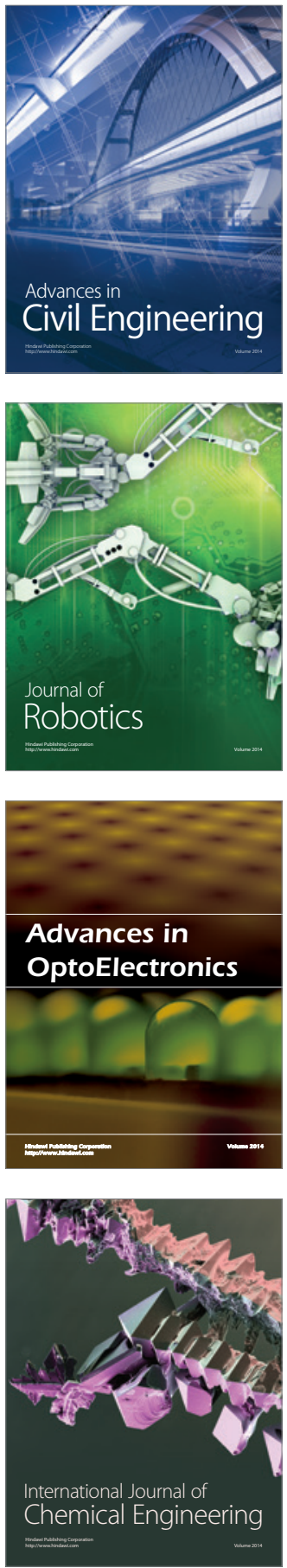

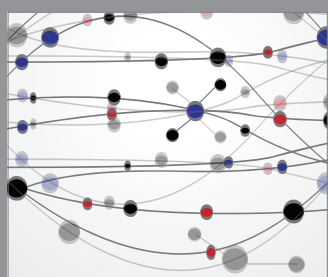

The Scientific World Journal

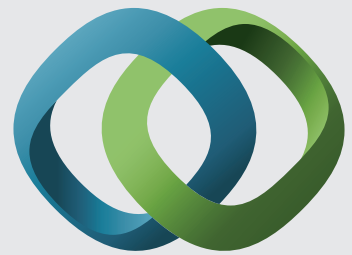

\section{Hindawi}

Submit your manuscripts at

https://www.hindawi.com
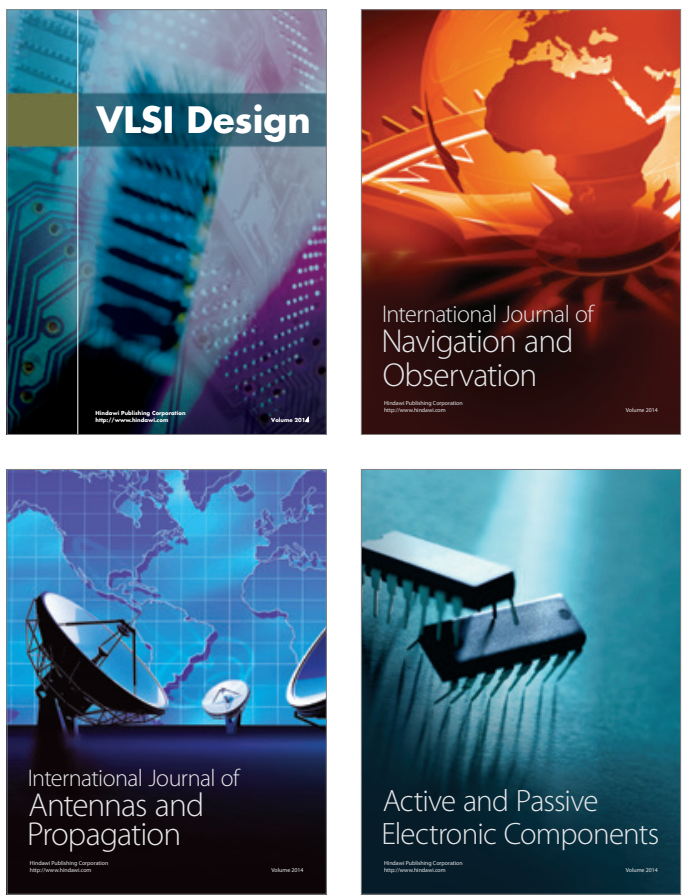
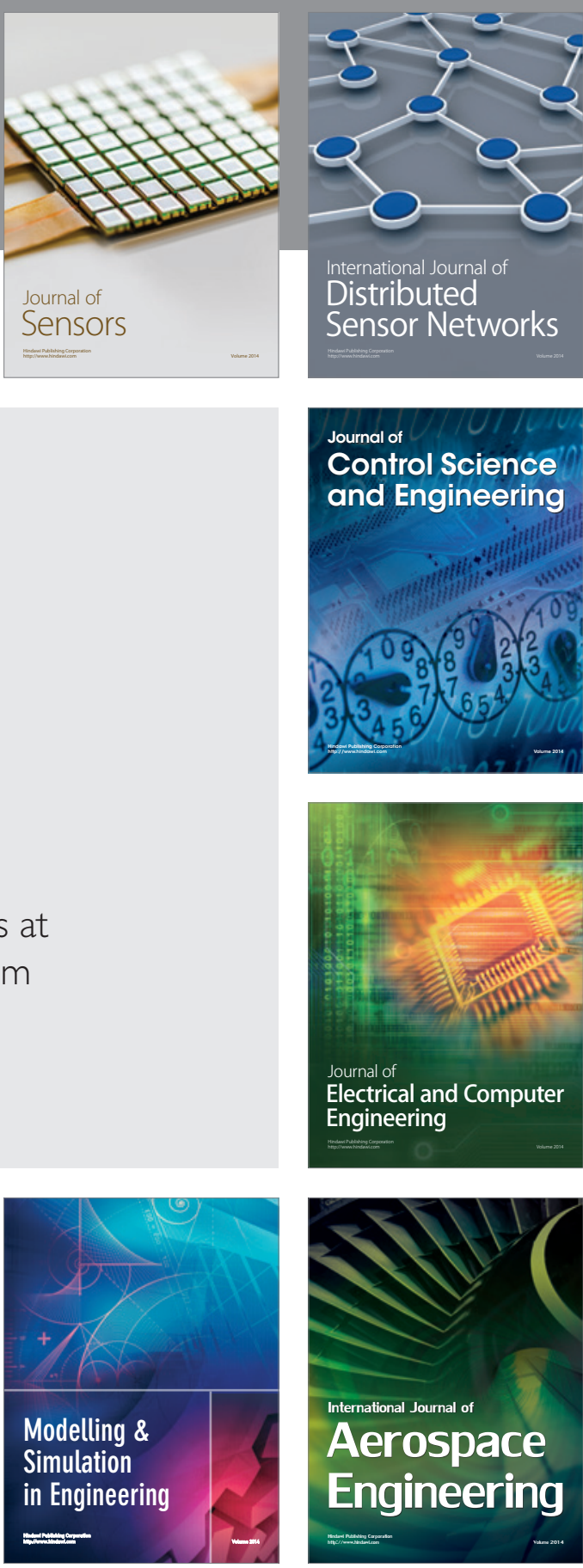

International Journal of

Distributed

Sensor Networks

$-$

Joumal of

Control Science

and Engineering
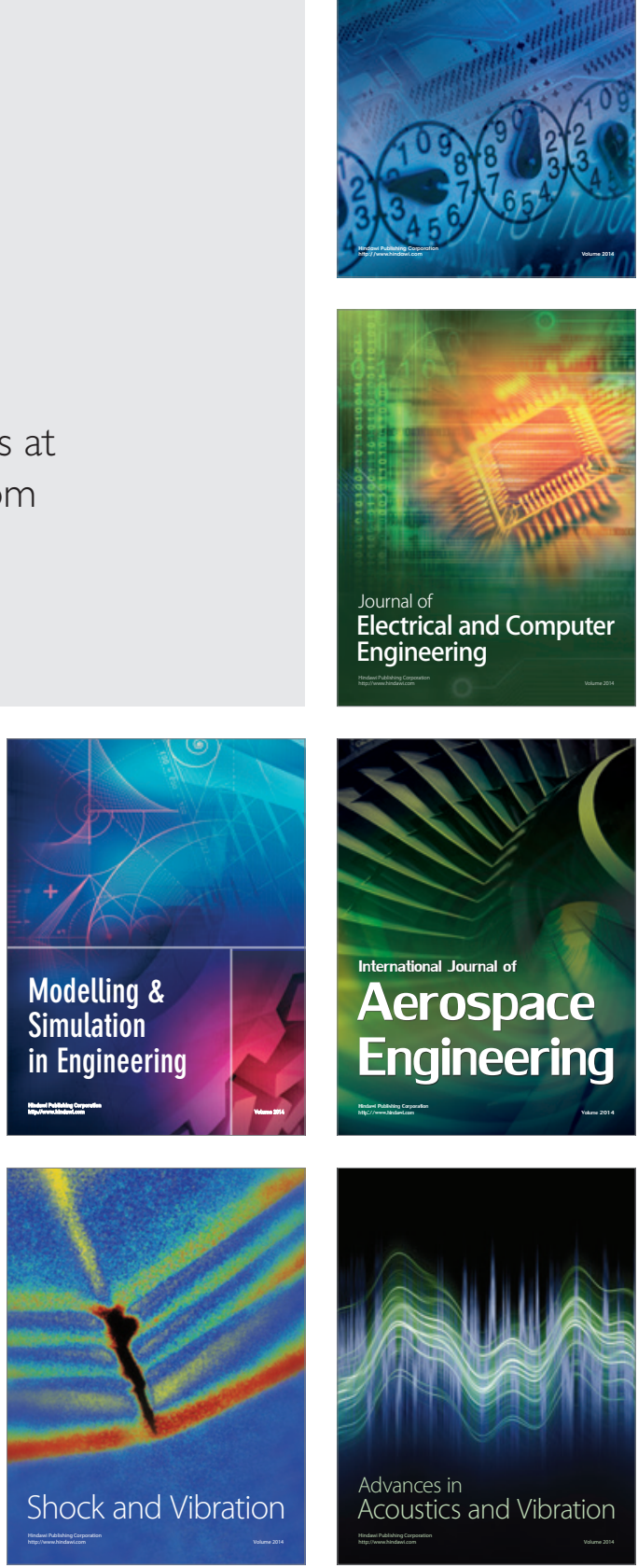